\title{
LINEAR TIME AND MEMORY-EFFICIENT COMPUTATION
}

\author{
KENNETH W. REGAN*
}

\begin{abstract}
A realistic model of computation called the Block Move (BM) model is developed. The BM regards computation as a sequence of finite transductions in memory, and operations are timed according to a memory cost parameter $\mu$. Unlike previous memory-cost models, the BM provides a rich theory of linear time, and in contrast to what is known for Turing machines, the BM is proved to be highly robust for linear time. Under a wide range of $\mu$ parameters, many forms of the BM model, ranging from a fixed-wordsize RAM down to a single finite automaton iterating itself on a single tape, are shown to simulate each other up to constant factors in running time. The BM is proved to enjoy efficient universal simulation, and to have a tight deterministic time hierarchy. Relationships among BM and TM time complexity classes are studied.
\end{abstract}

Key words. Computational complexity, theory of computation, machine models, Turing machines, random-access machines, simulation, memory hierarchies, finite automata, linear time, caching.

AMS/MOS classification: 68Q05,68Q10,68Q15,68Q68.

1. Introduction. This paper develops a new theory of linear-time computation. The Block Move (BM) model introduced here extends ideas and formalism from the Block Transfer (BT) model of Aggarwal, Chandra, and Snir [2]. The BT is a random access machine (RAM) with a special block transfer operation, together with a parameter $\mu: \mathbf{N} \rightarrow \mathbf{N}$ called a memory access cost function. The RAM's registers are indexed $0,1,2, \ldots$, and $\mu(a)$ denotes the cost of accessing register $a$. A block transfer has the form

$$
\text { copy }\left[a_{1} \ldots b_{1}\right] \text { into }\left[a_{2} \ldots b_{2}\right] \text {, }
$$

and is valid if these intervals have the same size $m$ and do not overlap. With regard to a particular $\mu$, the charge for the block transfer is $m+\mu(c)$ time units, where $c=\max \left\{a_{1}, b_{1}, a_{2}, b_{2}\right\}$. The idea is that after the initial charge of $\mu(a)$ for accessing the two blocks, a line of consecutive registers can be read or written at unit time per item. This is a reasonable reflection of how pipelining can hide memory latency, and accords with the behavior of physical memory devices (see [3], p1117, or [34], p 214). An earlier paper [1] studied a model called HMM which lacked the blocktransfer construct. The main memory cost functions treated in these papers are $\mu_{\log }(a):=\left\lceil\log _{2}(a+1)\right\rceil$, which reflects the time required to write down the memory address $a$, and the functions $\mu_{d}(a):=\left\lceil a^{1 / d}\right\rceil$ with $d=1,2,3, \ldots$, which model the asymptotic increase in communication time for memory laid out on a $d$-dimensional grid. (The cited papers write $f$ in place of $\mu$ and $\alpha$ for $1 / d$.) The two-level $I / O$ complexity model of Aggarwal and Vitter [3] has fixed block-size and a fixed cost for accessing the outer level, while the Uniform Memory Hierarchy (UMH) model of Alpern, Carter, and Feig [5] scales block-size and memory access cost upward in steps at higher levels.

The BM makes the following changes to the BT. First, the BM fixes the wordsize of the underlying machine, so that registers are essentially the same as cells on a Turing tape. Second, the BM provides native means of shuffling and reversing blocks.

* Department of Computer Science, State University of New York at Buffalo, 226 Bell Hall, Buffalo, NY 14620-2000. Supported in part by NSF Research Initiation Award CCR-9011248. 
Third and most important, the BM allows other finite transductions $S$ besides copy to be applied to the data in a block operation. A block move has the form

$$
S\left[a_{1} \ldots b_{1}\right] \text { into }\left[a_{2} \ldots b_{2}\right] \text {. }
$$

If $x$ is the string formed by the symbols in cells $a_{1}$ through $b_{1}$, this means that $S(x)$ is written to the tape beginning at cell $a_{2}$ in the direction of $b_{2}$, with the proviso that a blank $B$ appearing in the output $S(x)$ leaves the previous content of the target cell unchanged. This proviso implements shuffle, while reverse is handled by allowing $b_{1}<a_{1}$ and/or $b_{2}<a_{2}$. The block move is valid if the two intervals are disjoint, and meets the strict boundary condition if $S(x)$ neither overflows nor underflows $\left[a_{2} \ldots b_{2}\right]$. The work performed in the block move is defined to be the number $|x|$ of bits read, while the memory access charge is again $\mu(c), c=\max \left\{a_{1}, b_{1}, a_{2}, b_{2}\right\}$. The $\mu$-time is the sum of these two numbers. Adopting terms from [5], we call a BM $M$ memoryefficient if the total memory access charges stay within a constant factor (depending only on $M$ ) of the work performed, and parsimonious if the ratio of access charges to work approaches 0 as the input length $n$ increases.

In the BT model, Aggarwal, Chandra, and Snir [2] proved tight nonlinear lower bounds of $\Theta[n \log n]$ with $\mu=\mu_{1}, \Theta[n \log \log n]$ with $\mu=\mu_{d}, d>1$, and $\Theta\left[n \log ^{*} n\right]$ with $\mu=\mu_{\log }$, for the so-called "Touch Problem" of executing a sequence of operations during which every value in registers $R_{1} \ldots R_{n}$ is copied at least once to $R_{0}$. Since any access to $R_{a}$ is charged the same as copying $R_{a}$ to $R_{0}$, this gives lower bounds on the time for any BT computation that involves all of the input. In the BM model, however, the other finite transductions can glean information about the input in a way that copy cannot. Even under the highest cost function $\mu_{1}$ that we consider, many interesting nonregular languages and functions are computable in linear time.

Previous models. It has long been realized that the standard unit-cost RAM model $[21,31,18]$ is too powerful for many practical purposes. Feldman and Shapiro [22] contend that realistic models $\mathcal{M}$, both sequential and parallel, should have a property they call "polynomial vicinity" which we state as follows: Let $C$ be a data configuration, and let $H_{C}$ stand for the finite set of memory locations [or data items] designated as "scanned" in $C$. For all $t>0$, let $I_{t}$ denote the set of locations [or items] $i$ such that there exists an $\mathcal{M}$-program that, when started in configuration $C$, scans $i$ within $t$ time units. Then the model $\mathcal{M}$ has vicinity $v(t)$ if for all $C$ and $t$, $\left|I_{t}\right| /\left|H_{C}\right| \leq v(t)$. In 3D space, real machines "should have" at most cubic vicinity. The RAM model, however, has exponential vicinity even under the log-cost criterion advocated by Cook and Reckhow [18]. So do the random-access Turing machine (RAM-TM) forms described in [30, 26, 7, 14, 64], and TMs with tree-structured tapes (see $[57,63,51,52]$ ). Turing machines with $d$-dimensional tapes (see $[31,60,50]$ ) have vicinity $O\left(t^{d}\right)$, regardless of the number of such tapes or number of heads on each tape, even with head-to-head jumps allowed. The standard TM model, with $d=1$, has linear vicinity. The "RAM with polynomially compact memory" of Grandjean and Robson [29] limits integers $i$ that can be stored and registers $a$ that can be used to a polynomial in the running time $T$. This is not quite the same as polynomial vicinity - if $t \ll T$, the machine within $t$ steps could still address a number of registers that is exponential in $t$. The BM has polynomial vicinity under $\mu_{d}$ (though not under $\left.\mu_{\log }\right)$, because any access outside the first $t^{d}$ cells costs more than $t$ time units. The theorem of [56] that deterministic linear time on the standard TM (DLIN) is properly contained in nondeterministic TM linear time (NLIN) is not known to carry over to any model of super-linear vicinity. 
Practical motivations. The BM attempts to capture, with a minimum of added notation, several important properties of computations on real machines that the previous models neglect or treat too coarsely. The motivations are largely the same as those for the BT and UMH: As calibrated by $\mu$, memory falls into a hierarchy ranging from relatively small amounts of low-indexed fast memory up through to large amounts of slow external storage. An algorithm that enjoys good temporal locality of reference, meaning that long stretches of its operation use relatively few different data items, can be implemented as a BM program that first copies the needed items to low memory (figuratively, to a cache), and is rewarded by a lower sum of memory-access charges. Good spatial locality of reference, meaning that needed data items are stored in neighboring locations in approximately the order of their need, is rewarded by the possibility of batching or pipelining a sequence of operations in the same block move. However, the BM appears to emphasize the sequencing of data items within a block more than the BT and UMH do, and we speak more specifically of good serial access rather than spatial locality of reference. The BM breaks sequential computation into phases in which access is serial and the operation is a finite transduction, and allows "random" access only between phases. Both $\mu$-time $(n)$ and the count $R(n)$ of block moves provide ways to quantify random access as a resource. The latter also serves as a measure of parallel time, since finite transductions can be computed by parallel prefix sum. Indeed, the BM is similar to the Pratt-Stockmeyer vector machine [61], and can also be regarded as a fixed-wordsize analogue of Blelloch's "scan" model [11].

Results. The first main theorem is that the BM is a very robust model. Many diverse forms of the machine simulate each other up to constant factors in $\mu$-time, under a wide range of cost functions $\mu$. Allowing multiple tapes or heads, expanding or limiting the means of tape access, allowing invalid block moves, making block boundaries pre-set or data-dependent in a block move, even reducing the model down to a single finite automaton that iterates itself on a single tape, makes no or little difference. We claim that this is the first sweeping linear-time robustness result for a natural model of computation. A "linear speed-up" theorem, similar to the familiar one for Turing machines, makes the constant factors on these simulations as small as desired. All of this gives the complexity measure $\mu$-time a good degree of machineindependence. Some of the simulations preserve the work $(w)$ and memory-access charges $(\mu$-acc) separately, while others trade $w$ off against $\mu$-acc to preserve their sum.

Section 2 defines the basic BM model and also the reduced form. Section 3 defines all the richer forms, and Section 4 proves their equivalence. The linear speedup theorem and some results on memory-efficiency are in Section 5. The second main result of this paper, in Section 6, shows that like the RAM but unlike what is known for the standard multitape Turing machine model (see [36, 24]), the BM carries only a constant factor overhead for universal simulation. The universal BM given is efficient under any $\mu_{d}$, while separate constructions work for $\mu_{\log }$. In consequence, for any fixed $\mu=\mu_{d}$ or $\mu_{\log }$, the BM complexity classes $\mathrm{D} \mu \mathrm{TIME}[t]$ form a tight deterministic time hierarchy as the order of the time function $t$ increases. Whether there is any hierarchy at all when $\mu$ rather than $t$ varies is shown in Section 7 to tie back to older questions of determinism versus nondeterminism. This section also compares the BM to standard TM and RAM models, and studies BM complexity classes. Section 8 describes open problems, and Section 9 presents conclusions. 


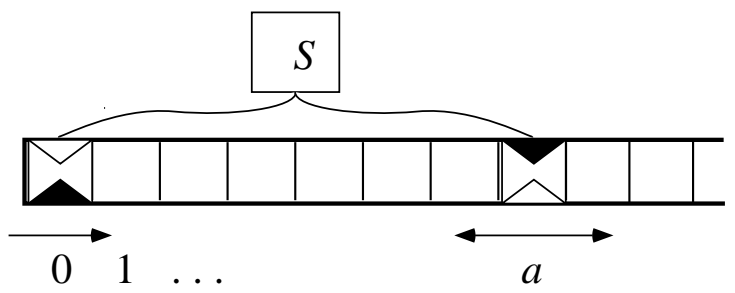

FIG. 1. BM with allowed head motions in a pass

2. The Block Move Model. We use $\lambda$ for the empty string and $B$ for the blank character. $\mathbf{N}$ stands for $\{0,1,2,3, \ldots\}$. Characters in a string $x$ of length $m$ are numbered $x_{0} x_{1} \cdots x_{m-1}$. We modify the generalized sequential machine (GSM) of [36] so that it can exit without reading all of its input.

DEFInition 2.1. A generalized sequential transducer (GST) is a machine $S$ with components $(Q, \Gamma, \delta, \rho, s, F)$, where $F \subseteq Q$ is the set of terminal states, $s \in Q \backslash F$ is the start state, $\delta:(Q \backslash F) \times \Gamma \rightarrow Q$ is the transition function, and $\rho:(Q \backslash F) \times \Gamma \rightarrow \Gamma^{*}$ is the output function. The $\mathrm{I} / \mathrm{O}$ alphabet $\Gamma$ may contain the blank $B$.

A sequence $\left(q_{0}, x_{0}, q_{1}, x_{1}, \ldots, q_{m-1}, x_{m-1}, q_{m}\right)$ is a halting trajectory of $S$ on input $x$ if $q_{0}=s, q_{m} \in F, x_{0} x_{1} \ldots x_{m-1}$ is an initial substring of $x$, and for $0 \leq i \leq m-$ $1, \delta\left(q_{i}, x_{i}\right)=q_{i+1}$. The output $S(x)$ is defined to be $\rho\left(q_{0}, x_{0}\right) \rho\left(q_{1}, x_{1}\right) \cdots \rho\left(q_{m-1}, x_{m-1}\right)$

By common abuse of notation we also write $S(\cdot)$ for the partial function computed by $S$. Except briefly in Section 8, all finite state machines we consider are deterministic. A symbol $c$ is an endmarker for a GST $S$ if every transition on $c$ sends $S$ to a terminal state. Without loss of generality, $B$ is an endmarker for all GSTs.

The intuitive picture of our model is a "circuit board" with GST "chips," each of which can process streams of data drawn from a single tape. The formalism is fairly close to that for Turing machines in [36].

Definition 2.2. A Block Machine $(B M)$ is denoted by $M=\left(\mathcal{Q}, \Sigma, \Gamma, \delta, B, S_{0}, F\right)$, where:

- $\mathcal{Q}$ is a finite set consisting of GSTs, move states, and halt states.

- $F$ is the set of halt states.

- Every GST has one of the four labels Ra, La, 0R, or $0 L$.

- Move states are labeled either $\lfloor a / 2\rfloor, 2 a$, or $2 a+1$.

- $\Sigma$ is the I/O alphabet of $M$, while the work alphabet $\Gamma$ is used by all GSTs.

- The start state $S_{0}$ is a GST with label Ra.

- The transition function $\delta$ is a mapping from $(\mathcal{Q} \backslash F) \times \Gamma$ to $\mathcal{Q}$.

We find it useful to regard GSTs as "states" in a BM machine diagram, reading the machine in terms of the specific functions they perform, and submerging the individual states of the GSTs onto a lower level. $M$ has two tape heads, called the "cell-0 head" and the "cell- $a$ head," which work as follows in a GST pass (Figure 1). Let $\sigma[i]$ stand for the symbol in tape cell $i$, and for $i, j \in \mathbf{N}$ with $j<i$ allowed, let $\sigma[i \ldots j]$ denote the string formed by the symbols from cell $i$ to cell $j$. 
Definition 2.3. A pass by a GST $S$ in a BM works as follows, with reference to the current address $a$ and each of the four modes $R a, L a, 0 R, 0 L$ :

(Ra) $S$ reads the tape moving rightward from cell $a$. Since $B$ is an endmarker for $S$, there is a cell $b \geq a$ in which $S$ exits. Let $x:=\sigma[a \ldots b]$ and $y:=S(x)$. If $y=\lambda$, the pass ends with no change in the tape. For $y \neq \lambda$, let $c:=$ $|y|-1$. Then $y$ is written into cells $[0 \ldots c]$, except that if $y_{i}=B$, cell $i$ is left unchanged. This completes the pass.

(La) $S$ reads the tape moving leftward from cell a. Unless $S$ runs off the left end of the tape (causing a "crash"), let $b \leq a$ be the cell in which $S$ exits. As before let $x:=\sigma[a \ldots b], y:=S(x)$, and if $y \neq \lambda, c:=|y|-1$. Then formally, for $0 \leq i \leq c$, if $y_{i} \neq B$ then $\sigma[i]:=y_{i}$, while if $y_{i}=B$ then $\sigma[i]$ is unchanged.

(0R) $S$ reads from cell 0, necessarily moving right. Let $c$ be the cell in which $S$ halts. Let $x:=\sigma[0 \ldots c], y:=S(x)$, and $b:=a+|y|-1$. Then $y$ is written rightward from a into cells $[a \ldots b]$, with the same convention about $B$ as above.

(0L) Same as $0 R$, except that $b:=a-|y|+1$, and $y$ is written leftward from a into $[a \ldots b]$.

Here $a, b$, and $c$ are the access points of the pass. Each of the four kinds of pass is valid if either (i) $y=\lambda$, (ii) $a, b, c \leq 1$, or (iii) $c<\min \{a, b\}$. The case $y=\lambda$ is called an empty pass, while if $|x|=1$, then it is called $a$ unit pass.

In terms of Section $1, R a$ and $L a$ execute the block move $S[a \ldots b]$ into $[0 \ldots c]$, except that the boundaries $b$ and $c$ are not set in advance and can depend on the data $x$. Similarly $0 R$ and $0 L$ execute $S[0 \ldots c]$ into $[a \ldots b]$. We make the distinction is that in a pass the read and write boundaries may depend on the data, while in a block move (formalized in the next section) they are set beforehand. The tape is regarded as linear for passes or block moves, but as a binary tree for addressing. The root of the tree is cell 1 , while cell 0 is an extra cell above the root. The validity condition says that the intervals $[a \ldots b]$ and $[0 \ldots c]$ must not overlap, with a technically convenient exception in case the whole pass is done in cells 0 and 1 . If a pass is invalid, $M$ is considered to "crash." A pass of type $R a$ or $L a$ figuratively "pulls" data to the left end of the tape, and we refer to it as a pull; similarly we call a pass of type $0 R$ or $0 L$ a put. Furthering the analogy to internal memory or to a processor cache, these pass types might be called a fetch and writeback, respectively. An $L a$ or $0 L$ pass can reverse a string on the tape.

Definition 2.4. A valid computation $\vec{c}$ by a $B M M=\left(\mathcal{Q}, \Sigma, \Gamma, \delta, B, S_{0}, F\right)$ is defined as follows. Initially $a=0$, the tape contains $x$ in cells $0 \ldots|x|-1$ with all other cells blank, and $S_{0}$ makes the first pass. When a pass by a GST $S$ ends, let c be the character read on the transition in which $S$ exited. Then control passes to $\delta(S, c)$. In a move state $q$, the new current address $a^{\prime}$ equals $\lfloor a / 2\rfloor, 2 a$, or $2 a+1$ according to the label of $q$, and letting $d$ be the character in cell $a^{\prime}$, control passes to state $\delta(q, d)$. All passes must be valid, and a valid computation ends when control passes to a halting state. Then the output, denoted by $M(x)$, is defined to be $\sigma[0 \ldots m-1]$, where $\sigma[m]$ is the leftmost non- $\Sigma$ character on the tape. If $M$ is regarded as an acceptor, then the language of strings accepted by $M$ is denoted by $L(M):=\{x \in$ $\Sigma^{*} \mid M(x)$ halts and outputs 1$\}$.

The convention on output is needed since a BM cannot erase, i.e. write B. Alternatively, for an acceptor, $F$ could be partitioned into states labeled ACCEPT and REJECT. 


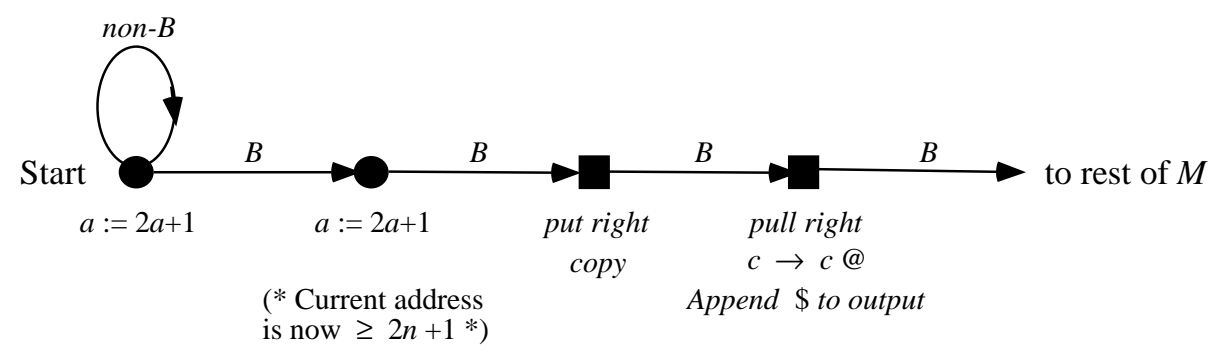

FIG. 2. A BM that makes a fresh track

Definition 2.5. A memory cost function is any function $\mu: \mathbf{N} \rightarrow \mathbf{N}$ with the properties (a) $\mu(0)=0$, (b) $(\forall a) \mu(a) \leq a$, and (c) $(\forall N \geq 1)(\forall a) \mu(N a) \leq N \mu(a)$.

Our results will only require the property $\left(\mathrm{c}^{\prime}\right):(\forall N \geq 1)\left(\exists N^{\prime} \geq 1\right)\left(\forall^{\infty} a\right) \mu(N a) \leq$ $N^{\prime} \mu(a)$. While property (c) can be named by saying that $\mu$ is "sub-linear," we do not know a standard mathematical name for $\left(c^{\prime}\right)$, and we prefer to call either $(c)$ or $\left(c^{\prime}\right)$ the tracking property for the following reason:

EXAMPLE 2.1. Tracking. Figure 2 diagrams a multichip BM routine that changes the input $x=x_{0} x_{1} \cdots x_{n-1}$ to $x_{0} @ x_{1} @ \cdots x_{n-2} @ x_{n-1} @ \$$, where @ acts as a "surrogate blank," and only @ or $B$ appears to the right of the $\$$. This divides the tape into two tracks of odd and even cells. A BM can write a string $y$ to the second track by pulling it as $B y_{0} B y_{1} \cdots B y_{m-1} B y_{m}$, since the blanks $B$ leave the contents of the first track undisturbed. Two strings can also be shuffled this way. Since $\mu(2 a) \leq 2 \mu(a)$, the tracking no more than doubles the memory access charges.

The principal memory cost functions we consider in this paper are the log-cost function $\mu_{\log }(a):=\left\lceil\log _{2}(a+1)\right\rceil$, and for all $d \geq 1$, the $d$-dimensional layout function $\mu_{d}(a):=\left\lceil a^{1 / d}\right\rceil$. These have the tracking property.

DEFINITION 2.6. For any memory cost function $\mu$, the $\mu$-time of a valid pass that reads $x$ and operates the cell-a head in the interval $[a \ldots b]$ is given by $\mu(a)+|x|+\mu(b)$. The work of the pass is $|x|$, and the memory access charge is $\mu(a)+\mu(b)$. A move state that changes a to $a^{\prime}$ performs 1 unit of work and has a memory access charge of $\mu(a)+\mu\left(a^{\prime}\right)$. The sum of the work over all passes in a valid computation $\vec{c}$ is denoted by $w(\vec{c})$, the total memory access charges by $\mu$-acc $(\vec{c})$, and the total $\mu$-time by $\mu(\vec{c}):=w(\vec{c})+\mu-a c c(\vec{c})$.

Intuitively, the charge for a pass is $\mu(a)$ time units to access cell $a$, plus $|x|$ time units for reading or writing the block, plus $\mu(b)$ to communicate to the CPU that the pass has ended and to re-set the heads. We did not write $\max \{\mu(a), \mu(b)\}$ because $b$ is not known until after the time to access $a$ has already been spent; this makes no difference up to a factor of two. Replacing $|x|$ by $|x|+|S(x)|$ or by $\max \{|x|,|S(x)|\}$, or adding $\mu(c)$ to $\mu(a)+\mu(b)$, also make no difference in defining $w$ or $\mu$-acc, this time up to a constant factor that may depend on $M$.

DeFINITION 2.7. For any input $x$ on which a BM $M$ has halting computation $\vec{c}$, we define the complexity measures

Work: $\quad w(M, x):=w(\vec{c})$.

Memory access: $\mu$-acc $(M, x):=\mu-a c c(\vec{c})$.

$\mu$-time: $\quad \mu$-time $(M, x):=w(M, x)+\mu$-acc $(M, x)$. 
Space: $\quad s(M, x):=$ the maximum of a for all access points a in $\vec{c}$. Pass count: $\quad R(M, x):=$ the total number of passes in $\vec{c}$.

$M$ is dropped when it is understood, and the above are extended in the usual manner to functions $w(n), \mu$-acc $(n), \mu$-time $(n), s(n)$, and $R(n)$ by taking the maximum over all inputs $x$ of length $n$. A measure of space closer to the standard TM space measure could be defined in the extended BM models of the next section by placing the input $x$ on a separate read-only input tape, but we do not pursue space complexity further in this paper. The pass count appears to be sandwiched between two measures of reversals for multitape Turing machines, namely the now-standard one of $[59,35,16]$, and the stricter notion of [43] which essentially counts keeping a TM head stationary as a reversal.

DEFINITION 2.8. For any memory cost function $\mu$ and recursive function $t$ : $\mathbf{N} \rightarrow \mathbf{N}, \mathrm{D} \mu \mathrm{TIME}[t]$ stands for the class of languages accepted by BMs $M$ that run in time $t(n)$, i.e. such that for all $x, \mu$-time $(M, x) \leq t(|x|)$. TLIN stands for $\mathrm{D} \mu_{1} \operatorname{TIME}[O(n)]$.

We also write $\mathrm{D} \mu \mathrm{TIME}[t]$ and TLIN for the corresponding function classes. Section 7 shows that TLIN is contained in the TM linear-time class DLIN. We argue that languages and functions in TLIN have true linear-time behavior even under the most constrained implementations.

We do not separate out the work performed from the total memory access charges in defining BM complexity classes, but do so in adapting the following notions and terms from [5] to the BM model.

DEFINITION 2.9.

(a) A BM $M$ is memory efficient, under a given memory cost function $\mu$, if there is a constant $K$ such that for all $x, \mu$-time $(M, x) \leq K \cdot w(M, x)$.

(b) $M$ is parsimonious under $\mu$ if $\mu$-time $(M, x) / w(M, x) \rightarrow 1$ as $|x| \rightarrow \infty$.

Equivalently, $M$ is memory efficient under $\mu$ if $\mu$ - $\operatorname{acc}(M, x)=O(w)$, and parsimonious under $\mu$ if $\mu$-acc $(M, x)=o(w)$, where the asymptotics are as $|x| \rightarrow \infty$. The intuition, also expressed in [5], is that efficient or parsimonious programs make good use of a memory cache.

Definition 2.9 does not imply that the given BM $M$ is optimal for the function $f$ it computes. Indeed, from Blum's speed-up theorem [12] and the fact that $\mu$-time is a complexity measure, there exist computable functions with no $\mu$-time optimal programs at all. To apply the concepts of memory efficiency and parsimony to languages and functions, we use the following relative criterion:

DEFINITION 2.10.

(a) A function $f$ is inherently $\mu$-efficient if for every $B M M_{0}$ that computes $f$, there is a $B M M_{1}$ which computes $f$ and a constant $K>0$ such that for all $x, \mu$-time $\left(M_{1}, x\right) \leq K \cdot w\left(M_{0}, x\right)$.

(b) $f$ is inherently $\mu$-parsimonious if for every $B M M_{0}$ computing $f$ there is a $B M M_{1}$ computing $f$ such that $\lim \sup _{|x| \rightarrow \infty} \mu$-time $\left(M_{1}, x\right) / w\left(M_{0}, x\right) \leq 1$.

By definition $\mu$-parsimony $\Longrightarrow \mu$-efficiency, and if $f$ is inherently efficient (resp. parsimonious) under $\mu_{1}$, then $f$ is inherently efficient (resp. parsimonious) under every memory cost function $\mu \leq \mu_{1}$.

Just for the next three examples, we drop the validity condition on rightward pulls; that is, we allow the tape intervals $[a \ldots b]$ and $[0 \ldots c]$ to overlap in an $R a$ 


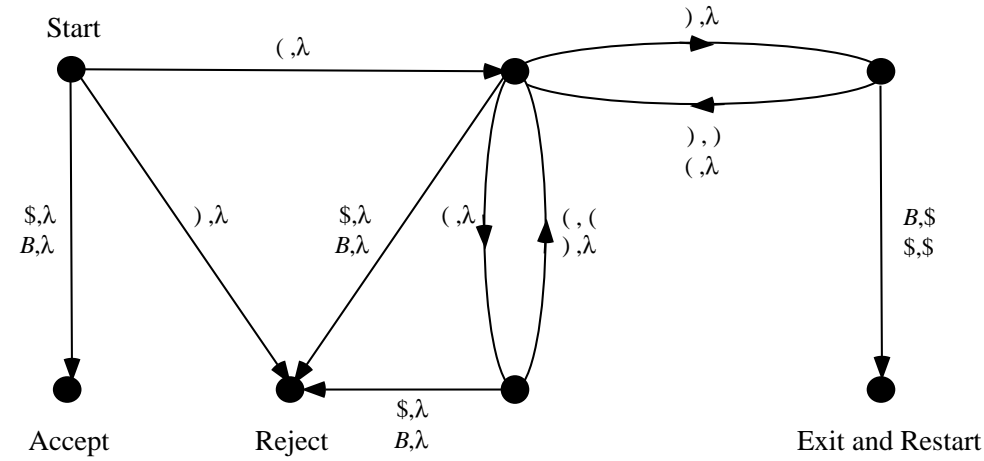

FIG. 3. Reduced-form BM for the language of balanced parentheses

move. This is intuitively reasonable so long as the cell-0 head does not overtake the cell- $a$ head and write over a cell that the latter hasn't read yet. Theorem 4.1 will allow us to drop the validity condition with impunity, but the proof of Theorem 2.12 below requires that it be in force.

Example 2.2. Balanced Parentheses. Let $D_{1}$ stand for the language of balanced parenthesis strings over $\Sigma:=\{()$,$\} . Let the GST S$ work as follows on any $x \in \Sigma^{*}$ : If $x=\lambda, S$ goes to a terminal state marked ACCEPT; if $x$ begins with ')', $S$ goes to REJECT. Else $S$ erases the leading '(' and thereafter takes bits in twos, translating

$$
((\mapsto(\quad)) \mapsto) \quad() \mapsto \lambda \quad)(\mapsto \lambda .
$$

If $x$ ends in '(' or $|x|$ is odd, $S$ also signals REJECT. Then $S$ has the property that for any $x \neq \lambda$ that it doesn't immediately reject, $x \in D_{1} \Longleftrightarrow S(x) \in D_{1}$. Furthermore, $|S(x)|<|x| / 2$. We can think of $D_{1}$ as being self-reducible in a particularly sharp sense.

Figure 3 shows the corresponding BM in the "reduced form" defined below. The '\$' endmarker is written on the first pass, and prevents leftover "garbage" on the tape from interfering with later passes. We take this for granted in some later descriptions of BMs. For any memory cost function $\mu$, the running time of $M$ is bounded by

$$
\sum_{i=0}^{\log _{2} n} \mu(0)+2^{i}+\mu\left(2^{i}\right)
$$

which is $O(n)$ even for $\mu=\mu_{1}$. Hence the language $D_{1}$ belongs to TLIN.

Example 2.3. Counting. Let $\Sigma:=\{\mathrm{a}, \mathrm{b}\}$. We can build a GST $S$ with alphabet $\Gamma=\{\mathrm{a}, \mathrm{b}, 0,1, \$, B\}$ that runs as follows on inputs of the form $x^{\prime}=x u \$$ with $x \in$ $\{\mathrm{a}, \mathrm{b}\}^{*}$ and $u \in\{0,1\}^{*}: S$ erases bits $x_{0}, x_{2}, x_{4}, \ldots$ of $x$ and remembers $|x|$ modulo 2. $S$ then copies $u$, and on reading the final $\$$ (or on the first pass, $B$ ), $S$ outputs $0 \$$ if $|x|$ was even, $1 \$$ if $|x|$ was odd. $S$ is also coded so that if $x=\lambda, S$ goes to Halt. Let $M$ be the BM which iterates $S$ on input $x$. Then $M(x)$ halts with $|x|$ in binary notation on its tape (followed by '\$' and "garbage"). The $\mu$-time for this iteration is likewise $O(n)$ even for $\mu=\mu_{1}$. 
EXAMPLE 2.4. Simulating a TM. Let $T:=\left(Q, \Sigma, \Gamma, \delta, B, q_{0}, F\right)$ be a single-tape TM in the notation of [36]. Define the $I D$ alphabet of $T$ to be $\Gamma_{I}:=(Q \times \Gamma) \cup \Gamma \cup\{\wedge, \$\}$, where $\wedge, \$ \notin \Gamma$. The simulating BM $M$ on an input $x=x_{0} \cdots x_{n-1}$ makes a rightward pull that lays down the delimited initial ID $\wedge\left(q_{0}, x_{0}\right) x_{1} x_{2} \cdots x_{n-1} \$$ of $T(x)$. The finite control of $T$ is turned into a single GST $S$ with alphabet $\Gamma_{I}$ that produces successive IDs in the computation with each pass. Whenever $T$ writes a blank, $M$ writes @. Let $T$ be programmed to move its head to cell 0 before halting. Then the final pass by $M$ removes the $\wedge$ and $\$$ and leaves exactly the output $y:=T(x)$ on the tape. Actually, because a BM cannot erase tape cells, $y$ would be followed by some number of symbols @, but Definition 2.4 still makes $y$ the output of $M$. Hence the BM is a universal model of computation.

The machines in Examples 2.2-2.4 only make rightward pulls from cell 0. Each is really a GST that iterates on its own output, a form generally known as a "cascading finite automaton" (CFA). Up to small technical differences, CFAs are comparable to the one-way "sweeping automata" studied by Ibarra et.al. [39, 41, 40, 37, 38, 15]. These papers characterize both one-way and two-way arrays of identical finite-state machines in terms of these and other automata and language classes. The following shows that the BM can be regarded as a generalization of these arrays, insofar as a $\mathrm{BM}$ can dynamically change its origin point $a$ and direction of operation.

DeFinition 2.11. The reduced form of the BM model consists of a single GST $S$ whose terminal states $q$ have labels $l_{1}(q) \in\{a,\lfloor a / 2\rfloor, 2 a, 2 a+1$, HALT $\}$ and $l_{2}(q) \in$ $\{R a, L a, 0 R, 0 L\}$. The initial pass has mode $R a$ with $a=0$. Whenever a pass by $S$ exits in some state $q$ with $l_{1}(q) \neq \mathrm{HALT}$, the labels $l_{1}(q)$ and $l_{2}(q)$ determine the address and mode for the next pass. Computations and complexity measures are defined as before.

THEOREM 2.12. Every $B M M$ is equivalent to a $B M M^{\prime}$ in reduced form, up to constant factors in all five measures of Definition 2.7.

Proof. The idea is to combine all the GSTs of $M$ into a single GST $S$ and save the current state of $M$ in cells 0 and 1 . Each pass of $M$ is simulated by at most six passes of $M^{\prime}$, except for a "staircase" of $O(\log n)$ moves at the end which is amortized into the constant factors. This simulation expands the alphabet but does not make any new tracks. The details are somewhat delicate, owing to the lack of internal memory when a pass by $M^{\prime}$ ends, and require the validity condition on passes. The full proof is in the Appendix.

In both directions, the tape cells used by $M$ and $M^{\prime}$ are almost exactly the same; i.e., $M$ is simulated "in place." Hence we consider the BM and the reduced form to be essentially identical. The idea of gathering all GSTs into one works with even less technical difficulty for the extended models in the next section.

3. Extensions of the BM. We consider five natural ways of varying the BM model: (1) Remove or circumvent the validity restriction on passes. (2) Provide "random addressing" rather than "tree access" in move states. (3) Provide delimiters $a_{1}, b_{1}, a_{2}, b_{2}$ for block moves $S\left[a_{1} \ldots b_{1}\right]$ into $\left[a_{2} \ldots b_{2}\right]$, where the cell $b_{1}$ in which $S$ exits is determined or calculated in advance. (4) Require that for every such block move, $b_{2}$ is such that $S(x)$ exactly fills $\left[a_{2} \ldots b_{2}\right]$. (5) Provide multiple main tapes and GSTs that can read from and write to $k$-many tapes at once. These extensions can be combined. We define them in greater detail, and in the next section, prove equivalences among them and the basic model. 
Definition 3.1. A BM with buffer mechanism has a new tape called the buffer tape, and GST chips $S$ with the following six labels and functions:

(RaB) The GST $S$ reads $x$ from the main tape beginning in cell a and writes $S(x)$ to the buffer tape. The output $S(x)$ must have no blanks in it, and it completely replaces any previous content of the buffer. Taking $b$ to be the cell in which $S$ exits, the $\mu$-time is $\mu(a)+|x|+\mu(b)$ as before.

(LaB) As for RaB, but reading leftward from cell a.

(BaR) Here $S$ draws its input $x$ from the buffer, and $S(x)$ is written on the main tape starting in cell a. Blanks in $S(x)$ are allowed and treated as before. When $S$ exits, even if it has not read all of the buffer tape, the buffer is flushed. With $b$ the destination of the last output bit (or $b=a$ if none), the $\mu$-time is likewise $\mu(a)+|x|+\mu(b)$.

(BaL) As for BaR, but writing $S(x)$ leftward from cell a.

(0B) As for RaB, but using the cell-0 head to read the input, and $\mu$-time $|x|+\mu(c)$.

(B0) As for BaR, but using the cell-0 head to write the output; likewise $\mu$-time $|x|+\mu(c)$.

All six types of pass are automatically valid. Further details of computations and complexity measures are the same as before. A BM with limited buffer mechanism has no GSTs with labels B0 or 0B, and consequently has no cell-0 head.

The original BM's moves of type $R a$ or $L a$ can now be simulated directly by $R a B$ or $L a B$ followed by $B 0$, while $0 R$ or $0 L$ is simulated by $0 B$ followed by $B a R$ or $B a L$. For the limited buffer mechanism the simulation is trickier, but for $\mu=\mu_{d}$ we will show that it can be done efficiently. The next extension allows "random access."

DeFINITION 3.2. The address mechanism adds an address tape and new load moves labeled RaA, LaA, and $0 A$. These behave and are timed like the buffer moves $R a B, L a B$, and $0 B$ respectively, but direct their output to the address tape instead. As with the buffer, the output completely replaces the previous content of the address tape. Addresses are written in binary notation with the least significant bit leftmost on the tape. The output $a^{\prime}$ of a load becomes the new current address. Move states may be discarded without loss of generality.

Example 3.1. Palindromes. Let $\mathrm{Pal}$ denote the language of palindromes over a given alphabet $\Sigma$. We sketch a BM $M$ with address mechanism that accepts Pal. On input $x, M$ makes a fresh track on its tape via Example 2.1, and runs the procedure of Example 2.3 to leave $n:=|x|$ in binary notation on this track. In running this procedure, we either exempt rightward pulls from the validity condition or give $M$ the buffer mechanism as well. The fresh-track cell which divides the right half of $x$ from the left half has address $n^{\prime}:=2\lfloor n / 2\rfloor+1$. A single $0 A$ move can read $n$ but copy the first bit as 1 to load the address $n^{\prime}$. $M$ then pokes a $\$$ into cell $n^{\prime}$. Another load prepends a ' 0 ' so as to address cell $2 n$, and $M$ then executes a leftward pull that interleaves the left half of $x$ with the right half. A bit-by-bit compare from cell 0 finishes the job. $M$ also runs in linear $\mu_{1}$-time.

The address mechanism provides for indirect addressing via a succession of loads, and makes it easy to implement pointers, linked lists, trees, and other data structures and common features of memory management on a BM, subject to charges for the number and size of the references.

Thus far, all models have allowed data-dependent block boundaries. We call any of the above kinds of BM $M$ self-delimiting if there is a sub-alphabet $\Gamma_{e}$ of endmarkers such that all GSTs in $M$ terminate precisely on reading an endmarker. 
(If we weaken this property slightly to allow a GST $S$ to exit on a non-endmarker on its second transition, then it is preserved in the proof of Theorem 2.12.) The remaining extensions pre-set the read block $\left[a_{1} \ldots b_{1}\right]$ and the write block $\left[a_{2} \ldots b_{2}\right]$, and this is when we speak of a block move rather than a pass. Having $b_{1}$ fixed would let us use the original GSM model from [36]. However, the machines that follow are always able to drop an endmarker into cell $b_{1}$ and force a GST $S$ to read all of $\left[a_{1} \ldots b_{1}\right]$. Hence we may ignore the distinction and retain 'GST' for consistency.

Definition 3.3. A block move is denoted by $S\left[a_{1} \ldots b_{1}\right]$ into $\left[a_{2} \ldots b_{2}\right]$ and has this effect on the tape: Let $x:=\sigma\left[a_{1} \ldots b_{1}\right]$. Then $S(x)$ is written to the tape beginning at $a_{2}$ and proceeding in the direction of $b_{2}$, with the proviso that each blank in $S(x)$ leaves the target cell unchanged, as in Definition 2.3. The block move is valid so long as the intervals $\left[a_{1} \ldots b_{1}\right]$ and $\left[a_{2} \ldots b_{2}\right]$ are disjoint. It underflows if $|S(x)|<\left|b_{2}-a_{2}\right|+1$, and overflows if $|S(x)|>\left|b_{2}-a_{2}\right|+1$.

By default we tolerate underflows and overflows in block moves. We draw an analogy between the next form of the BM and a text editor in which the user may mark a source and destination block and perform an operation on them. One important point is that the BM does not allow insertions and deletions of the familiar "cut-andpaste" kind; instead, the output flows over the destination block and overwrites or lets stand according to the use of $B$ in Definition 2.3. Willard [69] describes a model of a file system that lacks insertion and deletion, and gives fairly efficient algorithms for simulating them. Many text processors allow the user to define and move markers for points of immediate access in a file. Usually the maximum number of markers allowed is fixed to some number $m$. Adopting a term from data structures, we give the machine four fingers, with labels $a_{1}, b_{1}, a_{2}, b_{2}$, which can be assigned among the $m$ markers and which delimit the source and destination blocks in any block move. Finger $a_{1}$ may be thought of as the "cursor." The dual use of " $a_{1}$ " as the fixed label of a finger and as the number of the cell its assigned marker currently occupies may cause some confusion, but we try to keep the meanings clear below. The same applies to $a_{2}, b_{1}$, and $b_{2}$, and to later usage of these labels to name four special "address tapes."

Definition 3.4. A finger BM has four fingers labeled $a_{1}, b_{1}, a_{2}, b_{2}$, and some number $m \geq 4$ of markers. Initially one marker is on the last bit of the input, while all other markers and all four fingers are on the first bit in cell 0 . An invocation of a GST $S$ executes the block move $S\left[a_{1} \ldots b_{1}\right]$ into $\left[a_{2} \ldots b_{2}\right]$. The work performed by the block move is $\left|b_{1}-a_{1}\right|+1$, while the memory-access charge is $\mu(c)$, where $c=\max \left\{a_{1}, b_{1}, a_{2}, b_{2}\right\}$. In a move state, each marker on some cell a may be moved to cell $\lfloor a / 2\rfloor, 2 a$, or $2 a+1$ (or kept where it is), and the four fingers may be redistributed arbitrarily among the markers. The cost of a move state is the maximum of $\mu(a)$ over all addresses a involved in finger or marker movements; those remaining stationary are not charged.

One classical difference between "fingers" and "pointers" is that there is no fixed limit on the number of pointers a program can create. Rather than define a form of the BM analogous to the pointer machines of Schönhage and others [45, 66, 67, 49, 10], we move straight to a model that uses "random-access addressing," a mechanism usually considered stronger than pointers (for in-depth comparisons, see $[9,10]$ and also [68]). The following BM form is based on a random-access Turing machine (RAM-TM; cf. "RTM" in [30] and "indexing TM" in [14, 64, 8]), and is closest to the BT. 
Definition 3.5. A RAM-BM has one main tape, four address tapes, which are labeled $a_{1}, b_{1}, a_{2}, b_{2}$ and given their own heads, and a finite control comprised of RAMTM states and GST states. In a RAM-TM state, the current main-tape address a is given by the content of tape $a_{1}$. The machine may read and change both the character in cell $a$ and those scanned on the address tapes, and move each address tape head one cell left or right. In a GST state $S$, the address tapes give the block boundaries for the block move $S\left[a_{1} \ldots b_{1}\right]$ into $\left[a_{2} \ldots b_{2}\right]$ as described above, and control passes to some RAM-TM state. A RAM-TM step performs work 1 and incurs a memory-access charge of $\max \{\mu(a), \mu(b)\}$, where $b$ is the rightmost extent of an address tape head. Block moves are timed as above. Both a RAM-TM step and a block move add 1 to the pass count $R(n)$. Other details of computations are the same as for the basic BM model.

A fixed-wordsize analogue of the original BT model of [2] can now be had by making copy the only GST allowed in block moves. A RAM-BM with address loading can use block moves rather than RAM-TM steps to write addresses.

Definition 3.6. A finger BM or a RAM-BM obeys the strict boundary condition if in every block move $S\left[a_{1} \ldots b_{1}\right]$ into $\left[a_{2} \ldots b_{2}\right],|S(x)|$ equals $\left|b_{2}-a_{2}\right|+1$.

This constraint is notable when $S$ is such that $|S(x)|$ varies widely for different $x$ of the same length. The next is a catch-all for further extensions.

DeFinition 3.7. For $k \geq 2$, a $k$-input GST has $k$-many input tapes and one output tape, with $\delta:(Q \backslash F) \times \Gamma^{k} \rightarrow Q$ and $\rho:(Q \backslash F) \times \Gamma^{k} \rightarrow \Gamma^{*}$. Each input head advances one cell at each step.

Definition 3.8. A multitape BM has some number $k \geq 2$ of main tapes, each possibly equipped with its own address and/or buffer tapes, and uses $k$-input GSTs in passes or block moves.

Further details of computations and complexity measures for multitape BMs can be inferred from foregoing definitions, and various validity and boundary conditions can be formulated. The proofs in the next section will make the workings of these machines clear.

Finally, given two machines $M$ and $M^{\prime}$ of any kind and a cost function $\mu$, we say $M^{\prime}$ simulates $M$ linearly in $\mu$ if $\mu$-time $\left(M^{\prime}, x\right)=O(\mu$-time $(M, x))+O(|x|)$. The extra ' $O(n)$ ' is stated because like the RAM-TM, several BM variants give a sensible notion of computing in $s u b$-linear time, while all the simulations to come involve an $O(n)$-time preprocessing phase to set up tracks on the main tape. Now we can state:

Main Robustness Theorem 3.9. For any rational $d \geq 1$, all forms of the BM defined above simulate each other linearly in $\mu_{d}$-time.

If we adapted a standard convention for Turing machines to state that every BM on a given input $x$ takes time at least $|x|+1$ (cf. [36]), then we could say that all the simulations have constant-factor overheads in $\mu_{d}$-time.

4. Proof of the Main Robustness Theorem. The main problems solved in the proof are: (1) how to avoid overlaps in reading and writing by "tape-folding" (Theorem 4.1), (2) how to simulate random access with one read head whose movements are limited (Lemma 4.7), and (3) how to precompute block boundaries without losing efficiency (Lemma 4.13 through Theorem 4.17). Analogues of these problems are known in other areas of computation, but solving them with only a constant factor 
overhead in $\mu$-time requires some care. Some of the simulations give constant factor overheads in both $w$ and $\mu$-acc, but others trade off the work against the memory access charges. We also state bounds on $w^{\prime}$ and $\mu$-acc for the simulating machine $M^{\prime}$ individually, and on the number $R^{\prime}$ of passes $M^{\prime}$ requires, in or after proofs. The space $s^{\prime}(n)$ is always $O(s(n))$.

4.1. Simulations for data-dependent block boundaries. The first simulation uses the tracking property $\mu(N a) \leq N \mu(a)$ from Definition 2.5, and does not give constant-factor overheads in all measures. We give full details in this proof, in order to take reasonable shortcuts later.

THEOREM 4.1. For every BM $M$ with buffer there is a $B M M^{\prime}$ such that for every $\mu, M^{\prime}$ simulates $M$ linearly in $\mu$-time.

Proof. Let $M$ have the buffer mechanism. Let $C$ be the largest number of symbols output in any transition of any GST in $M$. Let $K:=\left\lceil\log _{2}(2 C+6)\right\rceil$ and $N:=2^{K}$. The BM $M^{\prime}$ first makes $N$-many tracks by iterating the procedure of Example 2.1. The track comprising cells $0, N, 2 N, 3 N, \ldots$ represents the main tape of $M$, while the two tracks flanking it are "marker tracks." The track through cells $2, N+2, \ldots$ represents the buffer tape. The other tracks are an "extension track," a "holding track," $C$-many "pull bays," and $C$-many "put bays." $M^{\prime}$ uses the symbol @ to reserve free space in tracks, and uses $\wedge$ and $\$$ to mark places in the tape. A $\$$ also delimits the buffer track so that leftover "garbage" does not interfere. Two invariants are that before every simulated pass by $M$ with current address $a$, the current address $a^{\prime}$ of $M^{\prime}$ equals $N a$, and the tracks apart from the main and buffer tracks contain only blanks and @ symbols.

The move $a:=2 a$ by $M$ is simulated directly by $a^{\prime}:=2 a^{\prime}$ in $M^{\prime}$. The move $a:=2 a+1$ is simulated by effecting $a^{\prime}:=\left\lfloor a^{\prime} / 2\right\rfloor K$-many times, then $a^{\prime}:=2 a^{\prime}+1$, and then $a^{\prime}:=2 a^{\prime} K$-many times. The move $a:=|a / 2|$ is simulated by effecting $a^{\prime}:=\left\lfloor a^{\prime} / 2\right\rfloor(K+1)$-many times, and then $a^{\prime}:=2 a K$-many times. Since $K$ is a constant, the overhead in $\mu$-acc for each move is constant. Henceforth we refer to "cell $a$ on the main track" in place of $a^{\prime}$.

We need only describe how $M^{\prime}$ simulates each of the six kinds of pass by $M$. Since $M$ has the $0 B$ and $B 0$ instructions, we may assume that the current address $a$ for the other four kinds is always $\geq 1$. For each state $q$ of a GST $S$ of $M, M^{\prime}$ has a GST $S_{q}^{\prime}$ which simulates $S$ starting in state $q$, and which exits only on the endmarker $\$$. We write just $S^{\prime}$ when $q=s$ or $q$ is understood.

(a) $R a B . M^{\prime}$ chooses $a_{1}:=2 a$, pokes $\wedge$ to the left of cell $a$, and pokes $\$$ to the left of cell $a_{1}$. $M^{\prime}$ then pulls $y_{1}:=S^{\prime}\left[a \ldots a_{1}-1\right]$ to the $C$-many pull bays. By the choice of $C,\left|y_{1}\right| \leq C a$, and so the pull is valid.

If the cell $b$ in which $S$ exits falls in the interval $\left[a \ldots a_{1}-1\right]$, then $S^{\prime}$ likewise exits in cell $b$. Since the exit character has no $\$$, the transition out of $S^{\prime}$ communicates that $S$ has exited. $M^{\prime}$ then makes $(K+1)$-many moves $a:=2 a$ so that $M^{\prime}$ now addresses cell $N a_{1}$ on the main track, which is cell $N^{2} a_{1}$ overall. $M^{\prime}$ puts $y:=y_{1}$ onto the extension track and then pulls $y$ onto the buffer track. One more put then overwrites the used portion of the extension track with @ symbols. $M^{\prime}$ then effects $a:=\lfloor a / 2\rfloor$ $(K+1)$-many times so that it addresses the original cell $a$ again, and re-simulates $S$ in order to overwrite the copy of $y$ on the pull bays by @ symbols. All of these passes are valid. $M^{\prime}$ finally removes the $\wedge$ and $\$$ markers at cells $a$ and $a_{1}$. The original time charge to $M$ was $\mu(a)+m+(b)$, where $m=b-a+1$. The time charged to $M^{\prime}$ in this case is bounded by: 


$$
\begin{array}{rlr}
\mu(N a) & +2+\mu(N a-1)+\mu\left(N a_{1}\right)+2+\mu\left(N a_{1}-1\right) & (\text { poke } \wedge \text { and } \$) \\
& +\mu(N a)+N m+\mu(N b) & (\text { simulate } S) \\
& +2 K \mu\left(N^{2} a_{1}\right) & \left(\text { move to cell } N a_{1}\right) \\
& +3 \mu\left(N^{2} a_{1}\right)+3 N^{2} m+3 \mu\left(N^{2} a_{1}+N^{2}(m-1)\right) & (\text { put and pull } y) \\
& +2 K \mu\left(N^{2} a_{1}\right)+\mu(N a)+N m+\mu(N b)+2 \mu(N a)+4+2 \mu\left(N a_{1}\right) \quad(\text { clean up) } \\
& \leq\left(14 N+8 N^{2} K+12 N^{2}\right) \mu(a)+\left(3 N^{2}+2 N\right) m+2 N \mu(b)+4 . & (m-1 \leq a)
\end{array}
$$

So far both the work $w^{\prime}$ and the memory access charges $\mu$-acc $c^{\prime}$ to $M^{\prime}$ are within a constant factor of the corresponding charges to $M$.

If $S$ does not exit in $\left[a \ldots a_{1}-1\right], S^{\prime}$ exits on the $\$$ marker. This tells $M^{\prime}$ to do a dummy pull to save the state $q$ that $S$ was in when $S^{\prime}$ hit the $\$$, and then to execute a put that copies $y_{1}$ from the pull bays to the put bays rightward from cell $a . M^{\prime}$ then effects $a:=2 a$ so now $a=a_{1}$, lets $a_{2}:=2 a_{1}$, pokes another $\$$ to the left of cell $a_{2}$, pulls $y_{2}:=S_{q}^{\prime}\left[a_{1} \ldots a_{2}-1\right]$ to the pull bays, and then puts $y_{2}$ into the put bays rightward of cell $a_{1}$. Since the $\$$ endmarker is in cell $N a_{1}-1$, this move is valid; nor does $y_{2}$ overlap $y_{1}$. If $S$ didn't halt in $\left[a_{1} \ldots a_{2}-1\right], M^{\prime}$ saves the state $q^{\prime}$ that $S$ was in when $S^{\prime}$ hit cell $a_{2}$, setting things up for the next stage with $a_{3}:=2 a_{2}$. The process is iterated until $S$ finally exits in some cell $b$ in some interval $\left[a_{j-1} \ldots a_{j}-1\right]$. Then $y:=y_{1} y_{2} \cdots y_{j}$ equals $S[a \ldots b] . \quad M^{\prime}$ moves to cell $N a_{j}$, puts $y$ onto the extension track rightward of cell $N a_{j}$, pulls $y$ to the buffer track, and "cleans up" the extension track as before. $M^{\prime}$ then takes $(K+1)$-many steps backward to cell $a_{j-1}$ and cleans up the pull and put bays with a pull and a put. Finally $M^{\prime}$ effects $a:=\lfloor a / 2\rfloor$ until it finds the $\wedge$ originally placed at cell $a$, meanwhile removing all of the $\$$ markers, and then removes the $\wedge$. This completes the simulated pull by $S$.

Let $j$ be such that $a_{j} \leq b<a_{j+1}$. Then the number $m$ of symbols read by $S$ is at least $a_{j}-a$. An induction on $j$ shows that the running totals of both $w^{\prime}$ and $\mu$-acc stay bounded by $D m$, where $D$ is a constant that depends only on $M$, not on $a$ or $j$. Hence the $\mu$-time for the simulation by $M^{\prime}$ is within $2 D$ times the $\mu$-time charged to $M$ for the pass. (However, when $j>0, \mu-a c c^{\prime} / \mu$-acc may no longer be bounded by a constant.)

(b) $0 B . M^{\prime}$ first runs $S$ on cell 0 only and stores the output $y_{0}$ on the first cells of the $C$-many put bays. $M^{\prime}$ then follows the procedure for $R a B$ with $a=1$. The analysis is essentially the same.

(c) $L a B . M^{\prime}$ first pokes a $\wedge$ to the left of cell $a$ and $\$$ to the left of cell $\lfloor a / 2\rfloor$. The $\wedge$ allows $M^{\prime}$ to detect whether $a$ is even or odd; i.e., whether it needs to simulate $a:=2 a$ or $a:=2 a+1$ to recover cell $a . M^{\prime}$ then pulls $y_{1}:=S^{\prime}[a \ldots\lfloor a / 2\rfloor]$ to the pull bays. Note that cell $\lfloor a / 2\rfloor$ is included; $M^{\prime}$ avoids a crash by remembering the first $2 C$-many symbols of $y_{1}$ in its finite control. If $S$ didn't exit in $[a \ldots\lfloor a / 2\rfloor], M^{\prime}$ remembers the state $q$ that $S$ would have gone to after processing cell $\lfloor a / 2\rfloor . M^{\prime}$ then copies cells $[0 \ldots\lfloor a / 2\rfloor-1]$ of the main track into cells $[\lfloor a / 2\rfloor+1 \ldots a]$ of the holding track, and does a leftward pull by $S_{q}^{\prime}$ to finish the work by $S$, stashing its output $y_{2}$ on the put bays. If $S_{q}^{\prime}$ does not exit before hitting the $\$$, then $S$ ran off the left end of the tape and $M$ crashed. Let $y:=y_{1} y_{2}$. Since $|y| \leq C a, M^{\prime}$ can copy $y$ to the buffer via cell $N a$ of the extension track by means similar to before, and "clean up" the pull and put bays and holding and extension tracks before returning control to cell $a$. Here both $w^{\prime}$ and $\mu$-acc' stay within a fixed constant factor of the corresponding charges to $M$ for the pass. 
(d) BaR. $M^{\prime}$ marks cell $a$ on the left with a $\$$, and does a dummy simulation of $S$ on cells $[0 \ldots a-1]$ of the buffer track. If $S$ exits in that interval, $M^{\prime}$ puts $S[0 \ldots a-1]$ directly onto the main track, and this completes the simulated pass. If not, $M^{\prime}$ puts $y_{0}:=S[0 \ldots a-1]$ onto the holding track rightward of cell $a$, and remembers the state $q$ in which $S^{\prime}$ hits the $\$ . M^{\prime}$ then follows the procedure for simulating $R a B$ beginning with $S_{q}^{\prime}$, except that it copies $@^{a} y_{0} y_{1} \cdots y_{j}$ to the extension track via cell $N a_{j}$. The final pull then goes to the main track but translates @ by $B$ so that the output written by $M$ lines up with cell $a$ of the main track. There is no need to "clean up" the read portion of the buffer tape since all writes to it are delimited. A calculation similar to that for $R a B$ yields a constant bound on the $\mu$-time and work for the simulated pass, though possibly not on the $\mu$-access charges.

(e) $B 0$. Under the simulation, this is the same as $0 B$ with the roles of the main track and buffer track reversed, and @ translated to $B$.

(f) BaL. $M^{\prime}$ marks cell $a$ on the left with $\$$ and puts $y_{1}:=S[0 \ldots a-1]$ rightward from cell $a$ of the holding track. If $S$ exits in that interval of the buffer tape, $M^{\prime}$ then pulls $y_{1}$ to the left end of the holding track. Note that if $\left|y_{1}\right|>a+1$ then $M$ was about to crash. $M^{\prime}$ remembers the first symbol $c^{\prime}$ of $y_{1}$ in its finite control to keep this last pull valid just in case $\left|y_{1}\right|=a+1$. Then $M^{\prime}$ puts $c^{\prime}$ into cell $a$, pokes a $\$$ to the left of cell $\lfloor a / 2\rfloor$, and executes a "delay-1 copy" of the holding track up to the $\$$ into the main track leftward from cell $a$. If a $B$ or @ is found on the holding track before the $\$$, meaning that $\left|y_{1}\right| \leq\lfloor a / 2\rfloor$, the copy stops there and the simulated $B a L$ move is finished. If not, i.e., if $\left|y_{1}\right|>\lfloor a / 2\rfloor$, then the delay allows the character $c^{\prime \prime}$ in cell $\lfloor a / 2\rfloor-1$ of the holding track to be suppressed when the $\$$ is hit, so that the copy is valid. Since $\left|y_{1}\right|>\lfloor a / 2\rfloor, M^{\prime}$ can now afford to do the following: poke a $\$$ to the right of cell $a$, effect $a:=2 a$, and do a leftward pull of cells $[2 a \ldots a+1]$ of the holding track into cells $[0 \ldots a-1]$ of the main track, translating @ as well as $B$ by $B$ to leave previous contents of the main track undisturbed. This stitches the rest of $y_{1}$ beginning with $c^{\prime \prime}$ correctly into place. $M^{\prime}$ also cleans up cells $[0 \ldots 2 a]$ of the holding track by methods seen before, and removes the $\$$ signs.

If $S$ does not exit in $[0 \ldots a-1], M^{\prime}$ executes a single $R a$ move starting $S^{\prime}$ from cell $a$, once again holding back the first character of this output $y_{2}$ just in case $y_{1}$ was empty and $\left|y_{2}\right|=a+1$. If this pull is invalid then likewise $\left|y_{2}\right|>a+1$ and $M$ crashed anyway. $M^{\prime}$ then concatenates $y_{2}$ to the string $y_{1}$ kept on the holding track to form $y$, and does the above with $y$. As in $L a B$, the overhead in both $w$ and $\mu$-acc is constant. This completes the proof. $\square$

The converse simulation of a BM by a BM with buffer is clear and has constantfactor overheads in all measures, by remarks following Definition 3.1. It is interesting to ask whether the above can be extended to a linear simulation of a concatenable buffer (cf. [46]), but this appears to run into problems related to the nonlinear lower bounds for the Touch Problem in [2]. The proof gives $w^{\prime}(n)=O(w(n)+n)$ and $R^{\prime}(n)=O(R(n) \log s(n))$. For $\mu-a c c^{\prime}$, the charges in the rightward moves are bounded by a constant times $\sum_{j=0}^{\log b} \mu\left(b / 2^{j}\right)$. For $\mu=\mu_{d}$ this sum is bounded by $2 d \mu_{d}(b)$, and this gives a constant-factor overhead on $\mu_{d^{-}}$acc. However, for $\mu=\mu_{\log }$ there is an extra factor of $\log b$.

COROLlary 4.2. A BM that violates the validity conditions on passes can be simulated linearly by a BM that observes the restrictions.

We digress briefly to show that allowing simultaneous read and overwrite on the main tape does not alter the power of the model, and that the convention on $B$ gives 
no power other than shuffle. A two-input Mealy Machine (2MM) is essentially the same as a 2-input GST with $\rho:(Q \backslash F) \times \Gamma^{2} \rightarrow \Gamma^{*}$.

Proposition 4.3. Let $M$ be a BM with the following extension to the buffer mechanism: in a put step, $M$ may invoke any $2 M M S$ that takes one input from the buffer and the other from the main tape, writes to the main tape, and halts when the buffer is exhausted. Then $M$ can be simulated by a BM $M^{\prime}$ with buffer at a constant-factor overhead in all measures, for all $\mu$.

Proof. To simulate the put by a $2 \mathrm{MM} S, M^{\prime}$ copies the buffer to a separate track so as to interleave characters with the segment of the main tape of $M$ concerned. Then $M^{\prime}$ invokes a GST $S^{\prime}$ that takes input symbols in twos and simulates $S$. Finally $M^{\prime}$ copies the output of $S^{\prime}$ from its own buffer over the main tape segment of $M$.D

Proposition 4.4. At a constant-factor overhead in all measures, for all $\mu$, a BM $M$ can be simulated by a $B M M^{\prime}$ that lacks $B$ but has the following implementation of shuffle: $M^{\prime}$ has the above buffer extension, but restricted to the fixed $2 M M$ which interleaves the symbols of its two input strings.

Proof. Let $\Gamma^{\prime}$ consist of $\Gamma$ together with all ordered pairs of characters from $\Gamma$; then the fixed $2 \mathrm{MM}$ can be regarded as mapping $\Gamma^{*} \times \Gamma^{*}$ onto $\Gamma^{\prime *}$. Now consider any GST $S$ of $M$ that can output blanks. Let $S^{\prime}$ write a dummy character @ in place of $B$, and let $M^{\prime}$ shuffle the output of $S^{\prime}$ with the content of the target block of the main tape. Finally $M^{\prime}$ executes a pass which, for all $c_{1}, c_{2} \in \Gamma$ with $c_{1} \neq @$, translates $\left(c_{1}, @\right)$ to $c_{1}$ and $\left(c_{1}, c_{2}\right)$ to $c_{2} . \square$

Besides the tracking property, our further simulations require something which, again for want of a standard mathematical name, we call the following:

Definition 4.5. A memory access cost function $\mu$ has the tape compression property if $(\forall \epsilon>0)(\exists \delta>0)\left(\forall^{\infty} a\right) \mu(\lceil\delta a\rceil)<\epsilon \mu(a)$.

Lemma 4.6. For any $d \geq 1$, the memory cost function $\mu_{d}$ has the tape compression property. In consequence, $\sum_{i=0}^{\log _{2} b} \mu_{d}\left(\left\lceil b / 2^{i}\right\rceil\right)=O\left(\mu_{d}(b)\right)$.

Proof. Take $\delta<\epsilon^{d}$. If $\delta$ of the form $1 / 2^{k}$ satisfies (a) for $\epsilon:=1 / 2$, then by elementary calculation, for all but finitely many $b, \sum_{i=0}^{\log _{2} b} \mu\left(\left\lceil b / 2^{i}\right\rceil\right) \leq 2 k \mu(b)$.

Lemma 4.6 promises a constant-factor overhead on the memory-access charges for "staircases" under $\mu_{d}$, whereas an extra log factor can arise under $\mu_{\log }$. The simulation of random access by tree access in the next lemma is the lone obstacle to extending the results that follow to $\mu_{\log }$. Since any function $\mu(m)$ with the tape compression property must be $\Omega\left[m^{\epsilon}\right]$ for some $\epsilon>0$, this pretty much narrows the field to the functions $\mu_{d}$. To picture the tree we write Up, Down LefT, and Down RIGHT in place of the moves $\lfloor a / 2\rfloor, 2 a$, and $2 a+1$ by $M^{\prime}$.

LEMMA 4.7. For every BM $M$ with address mechanism, there is a basic BM $M^{\prime}$ such that for all $d \geq 1, M^{\prime}$ simulates $M$ linearly under $\mu_{d}$.

Proof. We need to show how $M^{\prime}$ simulates a load step of $M$ that loads an address $a_{1}$ from cells $\left[a_{0} \ldots b_{0}\right]$ of the main tape. Let $m:=\left|a_{0}-b_{0}\right|+1 . M^{\prime}$ makes one spare track for operations on addresses. $M^{\prime}$ first pulls $a_{1}$ in binary to the left end of this track. By Theorem 4.1 we may suppose that this pull is valid. The cost is proportional to the charge of $\mu\left(a_{0}\right)+m+\mu\left(b_{0}\right)$ to $M$ for the load. By our convention on addresses, the least significant bit of $a_{1}$ is leftmost. In this pull, $M^{\prime}$ replaces the 
most significant ' 1 ' bit of $a_{1}$ by a ' $\$$ ' endmarker. $M^{\prime}$ then moves UP until its cell- $a$ head reaches cell 1 . With $k:=\left\lceil\log _{2} a_{0}\right\rceil$, the total memory access charges so far are proportional to $\sum_{i=0}^{k} \mu\left(2^{i}\right)$, which is bounded by a fixed constant times $\mu\left(a_{0}\right)$ by Lemma 4.6. Since the number of bits in $a_{1}$ is bounded by $C m$, where $C$ depends only on $M$, the work done by $M^{\prime}$ is bounded by $2 C m+k$. Since $k<\mu\left(a_{0}\right)$, we can ignore $k$. Hence the $\mu$-time charged so far to $M^{\prime}$ is bounded by a fixed constant of that charged to $M$ for the load.

$M^{\prime}$ now executes a rightward pull that copies all but the bit $b$ before the $\$$ endmarker, $b$ being the second most significant bit of $a_{1}$. This pull is not valid owing to an overlap on the fresh track, but by Corollary 4.2 we may suppose that it is valid. If $b=0 M^{\prime}$ moves Down LefT, while if $b=1, M^{\prime}$ moves Down Right. $M^{\prime}$ then executes a put that copies the remainder of $a_{1}$ (plus the $\$$ ) rightward from the new location $a . M^{\prime}$ iterates this process until all bits of $a_{1}$ are exhausted. At the end, $a=a_{1}$. Because of the tracking, $M^{\prime}$ moves Down LEFT once more so that it scans cell $2 a$, which is cell $a$ of the main track. This completes the simulated load. Recalling $\left|a_{1}\right| \leq C m$, and taking $l:=\left\lceil\log _{2}\left(a_{1}\right)\right\rceil$, the $\mu$-time for this second part is bounded by a constant times

$$
\sum_{i=0}^{l} \mu\left(2^{i}\right)+2(m-i)+\mu\left(2^{i}+2(m-i)\right) .
$$

By Lemma 4.6, the total memory access charges in this sum are bounded by a fixed constant times $\mu\left(a_{1}\right)$. The work to simulate the load is proportional to $m \cdot l$, that is, to $\left(\log a_{1}\right)^{2}$, which causes an extra $\log$ factor over the work by $M$ in the load. The key point, however, is that since $M$ loaded the address $a_{1}, M$ will be charged $\mu_{d}\left(a_{1}\right)$ on the next pass, which is asymptotically greater than $\left(\log a_{1}\right)^{2}$. Hence the $\mu_{d}$-time of $M^{\prime}$ stays proportional to the $\mu_{d}$-time of $M . \square$

Corollary 4.8. For every BM $M$ with both the address and buffer mechanism, we can find a basic $B M M^{\prime}$, and a $B M M^{\prime \prime}$ with the limited buffer mechanism, such that for any $d \geq 1, M^{\prime}$ and $M^{\prime \prime}$ simulate $M$ linearly under $\mu_{d}$.

Proof. The constructions of Lemma 4.7 and Theorem 4.1 yield $M^{\prime}$. For $M^{\prime \prime}$, we may first suppose that $M$ is modified so that whenever $M$ loads an address $a$, it first stores a spare copy of $a$ at the left end of a special track. Now consider a pass of type $B 0$ or $0 B$ made by $M . M^{\prime \prime}$ invokes a GST that remembers cell 0 and writes 1 to the address tape. Then with $a^{\prime}=1, M^{\prime \prime}$ simulates the pass by a $B a^{\prime} R$ or $R a^{\prime} B$ move. $M^{\prime \prime}$ then recovers the original address $a$ by loading it from the track. Thus far $M^{\prime \prime}$ is a BM with address and buffer that doesn't use its cell 0 head. The method of Lemma 4.7 then removes the address mechanism in a way unaffected by the presence of the buffer.

We remark that Lemma 4.7 and Theorem 4.1 apply to different kinds of pass by $M$, with two exceptions: First, pulling $a_{1}$ to the left end of the track in the proof of Lemma 4.7 may require simulating a buffer. However, this can be accounted against the cost to $M$ for the load. Second, the buffer is needed for overlaps in the further processing of $a_{1}$. However, this is needed for at most $O\left(\log \log \left(a_{1}\right)\right)$ many passes, each of which involves $O\left(\log a_{1}\right)$ work, and these costs are dominated by the time to process $a_{1}$ itself. Hence in Corollary 4.8 the bounds from Lemma 4.7 and Theorem 4.1 are additive rather than compounded, and with $\mu=\mu_{d}$ we obtain for $M^{\prime}, \mu_{d^{-}} a c c^{\prime}(n)=O\left(\mu_{d^{-}} a c c(n)\right), \mu_{\log ^{-}} a c c^{\prime}(n)=O\left(\mu_{\log ^{-}} a c c(n) \log s(n)\right)$, $w^{\prime}(n)=O(w(n)+n+R(n) \log s(n))$, and $R^{\prime}(n)=O(R(n) \log s(n))$. 
LEMma 4.9. For every RAM-BM $M$, we can find a $B M M^{\prime}$ with the address and buffer mechanisms, such that for any memory cost function $\mu$ that is $\Omega(\log n), M^{\prime}$ simulates $M$ linearly under $\mu$.

Proof. First, $M^{\prime}$ makes separate tracks for the address tapes and worktapes of $M$, and also for storing the locations of the heads on these tapes of $M$. Whenever $M$ begins a block move $S\left[a_{1} \ldots b_{1}\right]$ into $\left[a_{2} \ldots b_{2}\right], M^{\prime}$ first computes the signs of $b_{1}-a_{1}$ and $b_{2}-a_{2}$, and remembers them in its finite control. $M^{\prime}$ then loads the true address of cell $a_{1}$ on the main tape, and pulls the data through a copy of $S$ labeled $R a B$ or $L a B$ - depending on sign - to the buffer. Then $M^{\prime}$ loads 0 to access $a_{2}$, loads $a_{2}$ itself, and finally copies the buffer right or left from cell $a_{2}$. Since $\mu$ is $\Omega(\log n)$, the $\mu$-time charged to $M^{\prime}$ is bounded by a fixed constant times the charge of $1+\left|b_{1}-a_{1}\right|+\max \left\{\mu\left(a_{1}\right), \mu\left(a_{2}\right), \mu\left(b_{1}\right), \mu\left(b_{2}\right)\right\}$ incurred by $M$. Similarly the $\mu$-acc charge to $M^{\prime}$ has the same order as that to $M$, though if $\left|b_{1}-a_{1}\right|<\log \left(b_{1}\right)$, this may not be true of the work.

If $M$ executes a standard RAM-TM transition, the cost to $M$ is $1+\mu\left(a_{1}\right)+\mu(c)$, where $a_{1}$ is the cell addressed on the main tape and $c$ is the greatest extent of an address tape or worktape head of $M . M^{\prime}$ first loads $a_{1}$ and writes the symbol written by $M$ into location $a_{1}$ with a unit put. Then $M^{\prime}$ loads each of the addresses for the other tapes of $M$ in turn, updates each one with a unit pull and a unit put, remembers the head movement on that tape, and increments or decrements the corresponding address accordingly. The time charge for updating the other tapes stays within a fixed constant factor of $\mu(c)$. $\square$

Remark: It would be nice to have the last simulation work when the charge to $M$ for a RAM-TM transition is just $1+\mu\left(a_{1}\right)$. The difficulty is that even though $\left|a_{1}\right|<a_{1}$, it need not hold that $c<a_{1}$, since $M$ might be using a lot of space on its worktapes. The issue appears to come down to whether a multitape TM running in time $t$ can be simulated by a BM in $\mu$-time $O(t)$. We discuss related open problems in Section 8.

LEMma 4.10. A finger BM can be simulated by a BM with address and buffer mechanisms, with the same bounds as in Lemma 4.9.

Proof. $M^{\prime}$ stores and updates the finitely-many markers on separate tracks in a similar manner to the last proof. The extra work per block move simulated to write or load these addresses is $O(\log s(n))$ as before. Both here and in Lemma 4.9, $R^{\prime}(n)=O(R(n))$.

THEOREM 4.11. Let $M$ be a RAM-BM, a finger BM, or a BM with the address and/or buffer mechanisms. Then we can find a $B M M^{\prime}$ that simulates $M$ linearly under any $\mu_{d}$.

Proof. This follows by concatenating the constructions of the last two lemmas with that of Corollary 4.8. Since $R^{\prime}(n)=O(R(n))$ in the former, the bounds on work and pass count remain $w^{\prime}(n)=O(w(n)+n+R(n) \log s(n))$ and $R^{\prime}(n)=O(R(n) \log s(n))$.

This completes the simulation of most of the richer forms of the model by the basic BM, with a constant factor overhead in $\mu_{d}$-time. By similar means, one can reduce the number of markers in a finger BM all the way to four. In going up to the richer forms, we encounter the problem that the finger BM and RAM-BM have pre-set block boundaries for input, and if the strict boundary condition is enforced, also for output. 
4.2. Simulations for pre-set block boundaries. The simulation in Theorem 4.1 does not make $M^{\prime}$ self-delimiting because it does not predetermine the cell $b \in$ $\left[a_{0} \ldots a_{1}\right]$ in which its own simulating GST $S^{\prime}$ will exit. We could try forcing $S^{\prime}$ to read all of $\left[a_{0} \ldots a_{1}\right]$, but part (a) of the proof of Theorem 4.1 had $a_{1}:=2 a_{0}$, and if e.g. $\mu\left(a_{0}\right)=\sqrt{a_{0}}$ and $b-a$ is small, $M^{\prime}$ would do much more work than it should. However, if one chooses the initial increment $e$ to be too small in trying $a_{1}:=a_{0}+e$, $a_{2}:=a_{0}+2 e, a_{3}:=a_{0}+4 e \ldots$, the sum of the $\mu$-access charges may outstrip the work. To balance the charges we take $e:=\mu\left(a_{0}\right)$. This requires $M^{\prime}$ to calculate $\mu(a)$ dynamically during its computation, and involves a concept of "time-constructible function" similar to that defined for Turing machines in [36].

Definition 4.12. Let $\mu$ be a memory cost function, and let $t: \mathbf{N} \rightarrow \mathbf{N}$ be any function. Then $t$ is $\mu$-time constructible if $t(n)$ is computable in binary notation by a $B M M$ in $\mu$-time $O(t(n))$.

Note that the time is in terms of $n$, not the length of $n$. We use this definition for $t=\mu$ itself, in saying that $\mu$ is $\mu$-time constructible. The following takes $d$ to be rational because there are real numbers $d \geq 1$ such that no computable function whatever gives $\left\lceil m^{1 / d}\right\rceil$ to within a factor of 2 for all $m$. In this section it would suffice to estimate $\left\lceil m^{1 / d}\right\rceil$ by some binary number of bit-length $|m| / d$, but we need the proof idea of incrementing fingers and the exact calculation of $\mu_{d}(m)$ for later reference.

LEMMA 4.13. For any rational $d \geq 1$, the memory cost function $\mu_{d}$ is $\mu_{d}$-time constructible by a finger BM that observes the strict boundary condition.

Proof. For any rational $d \geq 1$, the function $\left\lceil m^{1 / d}\right\rceil$ is computable in polynomial time, hence in time $(\log m)^{O(1)}$ by a single-tape TM $T$. The finger BM $M$ simulates the tape of $T$ beginning in cell 2 , and tracks the head of $T$ with its "main marker" $m_{1}$. $M$ also uses a character @ which is combined into others like so: if $T$ scans some character $c$ in cell $a, M$ scans $(c, @)$. $M$ then uses two unit block moves $S[a \ldots a]$ into $[0 \ldots 0]$ and $S[0 \ldots 0]$ into $[a \ldots a]$ to read and write what $T$ does. It remains to simulate the head moves by $T$.

To picture a tree we again say UP, Down LEFT, and Down RIGHT in place of moves from $a$ to $\lfloor a / 2\rfloor, 2 a$, or $2 a+1$. $M$ can test whether $a$ is a left or right child by moving UP and DOWN LEFT and seeing whether the character scanned contains the @. If $T$ moves right and $a$ is a left child, $M$ then intersperses moves UP and Down RIGHT with unit block moves to and from cell 0 to change $(c, @)$ back to $c$ and place @ into cell $a+1$. If instead $a$ is a right child, $M$ introduces a new marker $m_{5}$ into cell 1 , and writes $\wedge$ there. $M$ moves $m_{5}$ Down LEFT to count how far UP $m_{1}$ has to go until it reaches either a left child or the root (i.e., cell 1). By unit block moves, $M$ carries@ along with $m_{1}$, and by assigning a finger to marker $m_{5}$, can test whether $m_{5}$ is on cell 1 . If $m_{1}$ reaches a left child, $M$ moves it UP, Down Right, and then Down LEFT until $m_{5}$ comes back to the $\wedge$. Then $m_{1}$ is in cell $a+1$. If $m_{1}$ hits the root marked by $\wedge$, then $a$ had the form $2^{k}-1$, and so $M$ moves $m_{1}$ Down LeFT $k$ times. The procedure for decrementing $m_{1}$ when $T$ moves left is similar, with RIGHT and LEFT reversed.

For each step by $T$, the work by $M$ is proportional to $\log a$. By Lemma 4.6 for $\mu_{d}$, the total memory-access charge for incrementing or decrementing a finger in cell $a$ is $O\left(\mu_{d}(a)\right)$. Since $a \leq(\log m)^{O(1)}$, the total $\mu_{d}$-time for the simulation is still a polynomial in $\log m$, and hence is $o\left(\mu_{d}(m)\right)$.

This procedure can also be carried out on one of $2^{K}$-many tracks in a larger machine, computing $a \pm 2^{K}$ instead of $a \pm 1$ to follow head moves by $T$. The counting 
idea of the next lemma resembles the linear-size circuits constructed for 0-1 sorting in [55].

LEMma 4.14. The function $\# \mathrm{a}(x)$, which gives the number of occurrences of ' $a$ ' in a string $x \in\{\mathrm{a}, \mathrm{b}\}^{*}$, is computable in linear $\mu_{1}$-time by a BM that observes the strict boundary condition.

Proof. The BM $M$ operates two GSTs $S_{1}$ and $S_{2}$ that read bits of $x$ in pairs. Each records the parity $p$ of the number of pairs 'ab' or 'ba' it has seen thus far, and if $|x|$ is odd, each behaves as though the input were $x \mathrm{~b} . S_{1}$ outputs the final value of $p$ to a second track. $S_{2}$ makes the following translations

$$
\mathrm{aa} \mapsto \mathrm{a} \quad \mathrm{bb} \mapsto \mathrm{b} \quad \mathrm{ab}, \mathrm{ba} \mapsto \begin{cases}\mathrm{b} & \text { if } p=0 \\ \mathrm{a} & \text { if } p=1\end{cases}
$$

to form a string $x^{\prime}$ such that $\left|x^{\prime}\right|=\lceil|x| / 2\rceil$. Then $\# \mathrm{a}(x)=2 \# \mathrm{a}\left(x^{\prime}\right)+p$. This is iterated until no a's are left in $x$, at which point the bits $p$ combine to form $\# \mathrm{a}(x)$ in binary notation with the least significant bit first.

$M$ begins with one marker $m_{1}$ in cell $n-1$. We first note that even setting up the two tracks requires a trick to get two more markers to cell $n-1 . M$ starts a marker $m_{5}$ in cell 1 and moves it Down LEFT or Down Right according to whether $m_{1}$ is on a left or right child. When $m_{1}$ reaches cell $1, m_{5}$ records $n-1$ in reverse binary notation. Then $M$ starts moving $m_{5}$ back up while ferrying $m_{2}, m_{3}$ along with $m_{1}$. Then $M$ places $m_{2}$ and $m_{3}$ into cells $2 n$ and $4 n-1$, and with reference to Example 2.1, executes $(c \mapsto c @)[0 \ldots n-1]$ into $[2 n \ldots 4 n-1]$ and copy $[2 n \ldots 4 n-1]$ into $[0 \ldots 2 n-1]$. This also uses one increment and decrement of a marker as in the proof of Lemma 4.13 .

$M$ uses a new marker $m_{6}$ to locate where the next bit $p$ will go, incrementing $m_{6}$ after running $S_{1}$. In running $S_{2}$, always $\left|S_{2}\left(x^{\prime}\right)\right|=\left\lceil\left|x^{\prime}\right| / 2\right\rceil$, and by appropriate parity tests using its markers $m_{1}, m_{2}$, and $m_{3}, M$ can place its fingers so that all these moves are valid and meet the strict boundary condition. For the $k$ th iteration by $S_{2}$, these three markers are all on cells with addresses lower than $n / 2^{k-2}$, and even if each needs to be incremented by 1 with the help of $m_{5}$, the $\mu_{1}$ charges for simulating the iteration still total less than a fixed constant times $n / 2^{k-2}$. This also subsumes the $O\left(\log ^{2} n\right)$ charge for updating $m_{6}$. Hence the sum over all iterations is still $O(n)$.

TheOREM 4.15. For every $B M M$ and rational $d \geq 1$, we can find a finger $B M$ $M^{\prime}$ that simulates $M$ linearly under $\mu_{d}$ and observes the strict boundary condition.

Proof. As in the proof of Theorem 4.1, let $C$ be the maximum number of characters output in any GST transition of $M$, and let $K:=\log _{2}(2 C+6) . M^{\prime}$ first makes $N:=2^{K}$ tracks, by using the last proof's modification of the procedure of Example 2.1. Besides $2 C$-many tracks for handling the output of passes and one track for the main tape of $M, M^{\prime}$ uses one track to record the current address $a$ of $M$ with the least significant bit rightmost, one to compute and store $e:=\mu_{d}(a)$ via Lemma 4.13, one to store addresses $a_{j}$ below, two for Lemma 4.14, and one for other arithmetic on addresses. $M^{\prime}$ uses eight markers. Marker $m_{1}$ occupies cell $N a$ to record the current address $a$ of $M$. A move to $\lfloor a / 2\rfloor$ by $M$ is handled by moving $m_{1}$ UP $K+1$ times and DOwn LEFT $K$ times, and other moves are handled similarly. Meanwhile, marker $m_{6}$ stays on the last bit of the stored address $a$, and updating $a$ requires only one marker increment or decrement and $O(\log \log a)$ work overall. From here on we suppress the distinction between $a$ and $N a$ and other details that are the same as in Theorem 4.1. 
First consider a rightward pull by $M$ that starts a GST $S$ from cell $a_{0}$ on its main tape. $M^{\prime}$ has already stored $a_{0}$ in binary, and computes $e:=\mu_{d}\left(a_{0}\right)$. Since $\mu_{d}\left(a_{0}\right) \leq a_{0}$, $e$ fits to the left of marker $m_{6}$ in cell $|a| . M^{\prime}$ then places $m_{3}$ into cell $|a|+1$ and $m_{4}$ into cell $2|a|+1$, and executes two block moves from $[|a| \ldots 0]$ and $[0 \ldots|a|]$ into $[|a|+1 \ldots 2|a|+1]$ that shuffle $a_{0}$ and $e$ on their respective tracks with the least significant bits aligned and leftmost. $M^{\prime}$ then executes add $[|a|+1 \ldots 2|a|+1]$ into $[|a| \ldots 0]$ to produce $a_{1}$. A final carry that would make $\left|a_{1}\right|>\left|a_{0}\right|$ and cause a crash can be caught and remembered in the finite control of $M^{\prime}$ by running a "dummy addition" first, and then marking cell $2|a|+1$ to suppress its output by the GST add. Then $M^{\prime}$ "walks" marker $m_{2}$ out to cell $a_{1}$ by using $m_{3}$ to read the value of $a_{1}$ and $m_{5}$ to increment $m_{3}$.

Next $M^{\prime}$ walks $m_{4}$ out to cell $e$ (i.e., $N e$ ), and keeps $m_{3}$ in cell 0 . Let $S^{\prime}$ be a copy of $S$ which pads the output of each transition out to length exactly $C$, and which sends its output $z$ to the $C$-many tracks used as "pull bays." $S^{\prime}$ is also coded so that if $S$ exits, $S^{\prime}$ records that fact and writes @ ${ }^{C}$ in each transition thereafter. Then $M^{\prime}$ can execute $S^{\prime}\left[a_{1} \ldots a_{2}\right]$ into $[0 \ldots e]$ in compliance with the strict boundary condition. Now $M^{\prime}$ can calculate the number $i$ of non-@ symbols in $z$ by the method of Lemma 4.14. To write the true output $y_{1}=S\left[a_{0} \ldots a_{1}\right]$ and ensure the block move is valid, $M^{\prime}$ must still use the pull bays to hold $y_{1}$, so $M^{\prime}$ calculates $i^{\prime}:=\lceil i / C\rceil$ (actually, $i^{\prime}=N\lceil i / C\rceil$ ). Next $M$ walks $m_{4}$ out to cell $i^{\prime}$, and can finally simulate the first segment of the pass by $S$ by executing $S\left[a_{1} \ldots a_{2}\right]$ into $\left[0 \ldots i^{\prime}\right]$.

If $S$ exited in $\left[a_{0} \ldots a_{1}\right], M^{\prime}$ need only transfer the output $y_{1}$ of the last pass onto the left end of the main track. This can be done in two block moves after locating markers into cells $i^{\prime}, C i^{\prime}$, and $2 C i^{\prime}$. Else, $M^{\prime}$ transfers $y_{1}$ instead to the put bays and assigns a new marker $m_{7}$ to the "stitch point" in the put bays for the next segment of $y$. The final marker $m_{8}$ goes to cell $a_{1}$ and is used for the left-end of the read block in all succeeding segments. In three block moves, $M^{\prime}$ can both double $e$ to $2 e$ and compute $a_{2}:=a_{0}+2 e$ using $a d d$ as before. If and when the current value of $e$ has length greater than $\left|a_{0}\right|, M^{\prime}$ reassigns marker $m_{6}$ to the end of $e$ rather than $a_{0}$, incrementing it each time $e$ is doubled. Then $M^{\prime}$ walks $m_{2}$ out to cell $a_{2}$ and, remembering the state $q$ of $S$ where the previous segment left off, produces $y_{2}:=S_{q}\left[a_{1}+1 \ldots a_{2}\right]$ by the same counting method as before. To stitch $y_{2}$ into place on the put bays, $M^{\prime}$ converts the current location of $m_{7}$ into a numeric value $k$, adds it to $i:=\left|y_{2}\right|$, and finds cells $i+k$ and $2 i+k$ for two block copies. In case $S$ did not exit in $\left[a_{1} \ldots a_{2}\right], m_{7}$ is moved to cell $i+k, m_{8}$ to $a_{2}, m_{2}$ to $a_{3}:=a_{0}+4 e$, and the process is repeated.

Let $b$ be the actual cell in which $S$ exits, and let $j \geq 0$ be such that $a_{j}<b \leq a_{j+1}$. Then the $\mu_{d}$-time charged to $M$ for the pull is at least

$$
t_{j}:=\mu_{d}\left(a_{0}\right)+\mu_{d}\left(a_{0}+2^{j-1} e\right)+2^{j-1} e \geq 2 e+2^{j-1} e .
$$

(For $j=0$, read " $2^{j-1 "}$ as zero.) By Lemma 4.6, the memory access charge for walking a marker out to cell $a_{j}$ is bounded by a constant (depending only on $d$ ) times $\mu_{d}\left(a_{j}\right)$. The charges for the marker arithmetic come to a polynomial in $\log a_{j}$, and the charges for stitching segments $y_{j}$ into place stay bounded by the work performed by $M^{\prime}$. Hence the $\mu_{d}$-time charged to $M^{\prime}$ is bounded by a constant times

$$
u_{j}:=\mu_{d}\left(a_{0}\right)+\sum_{i=0}^{j} \mu_{d}\left(a_{0}+2^{i} e\right)+e+\sum_{i=0}^{j-1} 2^{i} e .
$$

Then $u_{j} \leq e+\sum_{i=0}^{j} \mu_{d}\left(2^{i+1} a_{0}\right)+2^{j} e \leq 2^{j+2} e+2^{j} e \leq 10 t_{j}$. 
For a leftward pull step by $M, M^{\prime}$ uses the same choice of $e:=\mu_{d}\left(a_{0}\right)$. If $e>a_{0} / 2$, then $M^{\prime}$ just splits $\left[0 \ldots a_{0}\right]$ into halves as in the $(L a B)$ part of the proof of Theorem 4.1. Else, $M^{\prime}$ proceeds as before with $a_{j+1}:=a_{0}-2^{j} e$, and checks at each stage whether $a_{j+1} \geq a_{0} / 2$ so that the next simulated pull will be valid. If not, then the amount of work done by $M$ thus far, namely $2^{j-1} e$, is at least $a_{0} / 4$. Thus $M^{\prime}$ can copy all of $\left[a_{j} \ldots 0\right]$ to another part of the tape and finish it off while remaining within a constant factor of the charge to $M$. The remaining bounds are much the same as those for a rightward pull above.

For a rightward or leftward put, marker $m_{1}$ is kept at the current address $a$, cell 0 is remembered in the finite control, and the procedure for a rightward pull is begun with $a_{0}=1$ and $m_{8}$ assigned there. Here $e=1$, and the rest is a combination of the $(B a R)$ or $(B a L)$ parts of the proof of Theorem 4.1 to ensure validity, and the above ways to meet the strict boundary condition in all block moves. $\square$

Remarks: This simulation can be made uniform by providing $d$ as a separate input. It can also be done using 8 tracks rather than $2 C+6$, though even taking $e:=\mu_{d}\left(a_{0}\right) / C$ does not guarantee that the third stage of a rightward pull, which reads $\left[a_{0}+2 e, a_{0}+4 e\right]$, will be valid. The fix is first to write the strings $y_{j}$ further rightward on the tape, then assemble them at the left end. Theorem 4.15 preserves $w(n)+\mu_{d^{-}} a c c(n)$ up to constant factors, but doesn't do so for either $w(n)$ or $\mu_{d^{-}} a c c(n)$ separately. When $d<1$, the case $b=a$ gives a worst-case extra work of $a^{1 / d}$, while the case of $b=2 a$ gives a total memory access charge of roughly $2(\log a)(d-1) / d$ times $\mu_{d}(a)$. This translates into $w^{\prime}(n)=O\left(w(n)+n+R(n) s(n)^{1 / d}\right)$ and $\mu_{d^{-}} a c c^{\prime}(n)=$ $O\left(\mu_{d^{-}} a c c(n) \log s(n)\right)$. However, when $d=1$, both $w$ and $\mu_{1}-a c c$ are preserved up to a factor of $10 N$. Allowing that $\mu_{d}\left(a_{0}\right)$ can be estimated to within a constant factor in $O\left(\log a_{0}\right)$ block moves, the pass count still carries $R^{\prime}(n)=O\left(R(n) \log ^{2} s(n)\right)$ because each movement in walking a marker to $a_{j}$ adds 1 to $R^{\prime}$. The following shows some technical improvements of having addressing instead of tree access.

THEOREM 4.16. Let $\mu=\mu_{\log }$ or $\mu=\mu_{d}$ with d rational. Then every BM $M$ can be simulated linearly under $\mu$ by a RAM-BM $M^{\prime}$ with address loading that observes the strict boundary condition.

Proof. For $\mu_{d}$ the simulation of the finger BM $M^{\prime}$ from the last proof by a RAM-BM is clear - the RAM-BM can even use RAM-TM steps for the address arithmetic. For $\mu_{\log }$, the point is that $M^{\prime}$ can take $e:=\left|a_{0}\right|$, and we may presume $e$ is already stored. The calculated quantities $a_{j}$ can be loaded in one block move. (Using RAM-TM steps to write them would incur $\mu_{\log }$ access charges proportional to $\log a_{0} \log \log a_{0}$.) The tradeoff argument of the proof of Theorem 4.15 works even for $\mu_{\log }$, and the above takes care of a constant-factor bound on the other steps in the simulation. This also gives $R^{\prime}(n)=O(R(n) \log s(n))$.

The tradeoff method of Theorem 4.15 seems also to be needed for the following "tape-reduction theorem."

TheOREM 4.17. For every rational $d \geq 1$, a multitape BM $M$ can be simulated linearly in $\mu_{d}$-time by a one-tape $B M M^{\prime}$.

Proof. Suppose that $M$ uses $k$ tapes, each with its own buffer, and GSTs $S$ that produce $k$ output strings as well as read $k$ inputs. We first modify $M$ to a machine $M^{\prime}$ that has $k$ main tracks, $k$ address tracks, one "input track," and one "buffer track." For any pass by $M$ with $S, M^{\prime}$ will interleave the $k$ inputs on the input track, do one separate pull for each of the $k$ outputs of $S$, and interleave the outputs on its buffer 
track. When $M$ subsequently invokes a $k$-input GST $T$ to empty its buffers, $M^{\prime}$ uses a 1-tape GST that simulates $T$ on the buffer track, invoking it $k$ times to write each of the $k$ outputs of $T$ to their destinations on the main tracks.

It remains only to show how $M^{\prime}$ marks the portions of the inputs to interleave. As in the proof of Theorem 4.15, there is the difficulty of not knowing in advance how long $S$ will run on its $k$ inputs. The solution is the same. $M^{\prime}$ first calculates the maximum $a_{j}$ of the addresses $a_{1}, \ldots, a_{k}$ on its address tracks, and then calculates $e:=\mu_{d}\left(a_{j}\right)$. For each $i, 1 \leq i \leq k, M^{\prime}$ drops an endmarker into cell $a_{i} \pm e$ according to the direction on main track $i$. Then $M^{\prime}$ copies only the marked-off portions of the tracks, putting those on its input track, and simulates the one-tape version $S_{1}$ of $S$. If $S_{1}$ exits within that portion, then $M^{\prime}$ continues as $M^{\prime}$ does. If $S_{1}$ does not exit within that portion, $M^{\prime}$ tries again with $a_{i} \pm 2 e, a_{i} \pm 4 e, \ldots$ until it does. The same calculation as in Theorem 4.15, plus the observation that if the direction on track $j$ is leftward then no track uses an address greater than $2 a_{j}$, completes the proof.

Finally we may restate the Main Robustness Theorem 3.9 in a somewhat stronger form:

Theorem 4.18. For any rational $d \geq 1$, all of the models defined in Section 3 are equivalent, linearly in $\mu_{d}$-time, to a BM in reduced form that is self-delimiting with '\$? as its only endmarker.

Proof. This is accomplished by Theorems 2.12 through 4.17. The procedures of Lemmas 4.15 and 4.7 and Theorem 4.1 are self-delimiting, and need only one endmarker $\$$. The trick of writing $\$$ on special tracks into the cell immediately left or right of the addressed cell $a$ allows $\$$ to survive the proof of Theorem 2.12 without being "tupled" into the characters $c_{0}, c_{1}$, or $c_{a}$.

With all this said and verified, we feel justified in claiming that there is one salient Block Machine model, and that the formulations given here are natural. The basic $\mathrm{BM}$ is the tightest for investigating the structure of computations, and helps the lower bound technique we suggest in Section 8. The richer forms make it easier to show that certain functions do belong to $\mathrm{D} \mu_{d} \operatorname{TIME}[t(n)]$.

5. Linear Speed-Up and Efficiency. The following "linear speed-up" theorem shrinks the constants in all the above simulations, at the usual penalty in alphabet size. First we give a precise definition:

DEFinition 5.1. The linear speed-up property for a model of computation and measure of time complexity states that for every machine $M$ with running time $t(n)$, and every $\epsilon>0$, there is a machine $M^{\prime}$ that simulates $M$ and runs in time $\epsilon \cdot t(n)+$ $O(n)$.

In the corresponding definition for Turing machines in [36], the additive $O(n)$ term is $n+1$ and is used to read the input. For the DTM, time $O(n)$ properly contains time $n+1$, while for the NTM these are equal [13]. For the BM under cost function $\mu$, the $O(n)$ term is $n+\mu(n)$.

THEOREM 5.2. With respect to any unbounded memory cost function $\mu$ that has the tape compression property, all of the BM variants described in Sections 2 and 3 have the linear speed-up property.

Proof. Let the BM $M$ and $\epsilon>0$ be given. The BM $M^{\prime}$ uses two tracks to simulate the main tape of $M$. Let $\delta$ in the tape compression property be such that for almost 
all $n, \mu(\delta n) \leq(\epsilon / 12 C) \cdot \mu(n)$. Here $C$ is a constant that depends only on $M$. Let $k:=\lceil 1 / 2 \delta\rceil$, let '@' stand for the blank in $\Gamma$, and let $\Gamma^{\prime}:=\Gamma^{k} \cup\{B\} . M^{\prime}$ uses $B$ only to handle its own two tracks. We describe $M^{\prime}$ as though it has a buffer; the constant $C$ absorbs the overhead for simulating one if $M^{\prime}$ lacks the buffer mechanism. On any input $x$ of length $n, M^{\prime}$ first spends $O(n)$ time units on a pull step that writes $x$ into $\lceil n / k\rceil$-many characters over the compressed alphabet $\Gamma^{\prime}$ on the main track. Thereafter, $M^{\prime}$ simulates $M$ with compressed tapes. In any pass by $M$ that writes output to the main tape, $M^{\prime}$ writes the compressed output to the alternate track. $M^{\prime}$ then uses the pattern of @ symbols in each compressed output character to mask the elements of each main track character that should not be overwritten, sending the combined output to the buffer. One more pass writes the result back to the main tape. If the cost to $M$ for the pass was $\mu(a)+|b-a|+\mu(b)$, the cost to $M^{\prime}$, allowing for the tracking, is no more than

$$
\begin{gathered}
3[\mu(2\lceil a / k\rceil)+(2 / d)|b-a|+2+\mu(2\lceil b / k\rceil)] \\
\leq(\epsilon / 2) \mu(a)+(\epsilon / 2)|b-a|+6+(\epsilon / 2) \mu(b) .
\end{gathered}
$$

The ' +2 ' and ' +6 ' allow for an extra cell at either end of the compressed block. Since $\mu$ is unbounded, we have $\mu(a) \cdot(\epsilon / 2)+6 \leq \epsilon \cdot \mu(a)$ for all but finitely many $a$. The main technical difficulty of the standard proof for TMs is averted because $\mu$ absorbs any time that $M$ might spend moving back and forth across block boundaries. The compression by a factor of $\epsilon$ holds everywhere except for cells $1, \ldots, m$ on the main tape, where $m$ is least such that $\mu(m) \geq 12 / \epsilon$, but $M^{\prime}$ can keep the content of these cells in its finite control. The remaining details are left to the reader. For BMs with address tapes, we may suppose that the addresses are written in a machine-dependent radix rather than in binary.

Corollary 5.3. For all of the simulations in Theorems 2.12-4.17, and all $\epsilon>0$ :

(a) If $M$ runs in $\mu_{d}$-time $t(n)=\omega(n)$, then $M^{\prime}$ can be constructed to run in $\mu_{d}$-time $\epsilon t(n)$ for all but finitely many $n$.

(b) If $M$ runs in $\mu_{d}$-time $O(n)$, then $M^{\prime}$ can be made to run in $\mu_{d}$-time $(1+\epsilon) n$.

Mostly because of Lemma 4.7 and Theorem 4.15, the above simulations do not guarantee constant factor overheads in either $w$ or $\mu$-acc. They do, however, preserve $\mu$-efficiency.

Proposition 5.4. For all of the simulations of a machine $M$ by a machine $M^{\prime}$ in Theorems 2.12-4.17, and memory cost functions $\mu$ they hold for, if $M$ is $\mu$-efficient then $M^{\prime}$ is also $\mu$-efficient.

Proof. Let $K_{1}$ be the constant from the simulation of $M$ by $M^{\prime}$, and let $K_{2}$ come from Definition 2.9(a) for $M$. Then for all but finitely many inputs $x$, we have

$\mu$-time $\left(M^{\prime}, x\right) \leq K_{1}(\mu$-time $(M, x)+|x|) \leq K_{1}\left(K_{2}(w(M, x)+|x|) \leq 2 K_{1} K_{2} w\left(M^{\prime}, x\right)\right.$.

The last inequality follows because every simulation has $w\left(M^{\prime}, x\right) \geq w(M, x)$ and $w\left(M^{\prime}, x\right) \geq|x|$. Hence $M^{\prime}$ is $\mu$-efficient.

So long as we adopt the convention that every function takes work at least $n+1$ to compute, we can state:

COROLlary 5.5. For any memory cost function $\mu_{d}$, with $d \geq 1$ and rational, the notion of a language or function being memory efficient under $\mu_{d}$ does not depend on the choice among the above variants of the BM model. 
We do not have analogous results for parsimony. However, the above allows us to conclude that for $d=1,2,3, \ldots$, memory-efficiency under $\mu_{d}$ is a fundamental property of languages or functions. Likewise we have a robust notion of the class $\mathrm{D} \mu_{d} \operatorname{TIME}[t(n)]$ of functions computable in $\mu_{d}$-time $t(n)$, for any time bound $t(n) \geq n$. The next section shows that for any fixed $d$, the classes $\mathrm{D} \mu_{d} \operatorname{TIME}[t(n)]$ form a tight hierarchy as the time function $t$ varies.

6. Word Problems and Universal Simulation. We use a simple representation of a list $\vec{x}:=\left(x_{1}, \ldots, x_{m}\right)$ of nonempty strings in $\Sigma^{*}$ by the string $x_{1} \# \ldots \# x_{m} \#$, where $\# \notin \Sigma$. More precisely, we make the last symbol $c$ of each element a pair $(c, \#)$ so as to separate elements without adding space, and also use pair characters $(c, @)$ or $(c, \$)$ to mark selected elements. The size of the list is $m$, while the bit-length of the list is $n:=\sum_{i=1}^{m}\left|x_{i}\right|$. We let $r$ stand for $\max \left\{\left|x_{i}\right|: 1 \leq i \leq m\right\}$. Following [16] we call the list normal if the strings $x_{i}$ all have length $r$. We number lists beginning with $x_{1}$ to emphasize that the $x_{i}$ are not characters.

LEMMA 6.1.

(a) The function mark $(\vec{x}, y)$, which marks all occurrences of the string $y$ in the normal list $\vec{x}$, belongs to TLIN.

(b) The function shuffle, which is defined for normal lists $\vec{x}:=\left(x_{1}, \ldots, x_{m}\right)$ and $\vec{y}:=\left(y_{1}, \ldots, y_{m}\right)$ of the same length and element size $r$ by shuffle $(\vec{x}, \vec{y})=$ $\left(x_{1}, y_{1}, x_{2}, y_{2}, \ldots, x_{m}, y_{m}\right)$, belongs to TLIN. Here $r$ as well as $m$ may vary.

Remark: Even if the lists $\vec{x}$ and $\vec{y}$ are not normal, mark and shuffle can be computed in linear $\mu_{1}$-time so long as they are balanced in the sense that $(\exists k)(\forall i) 2^{k-1}<\left|x_{i}\right| \leq$ $2^{k}$. This is because a balanced list can be padded out to a normal list in linear $\mu_{1}$-time (we do not give the details here), and then the padding can be removed. To normalize an unbalanced list may expand the bit-length quadratically, and we do not know how to compute shuffle in linear $\mu_{1}$-time for general lists.

Proof. (a) Let $r$ be the element size of the normal list $\vec{x}$. If $|y| \neq r$, then there is nothing to do. Else, the BM $M$ uses the idea of "recursive doubling" (cf. the section on vector machines in [6]) to produce $y^{k}$, where $k=\left\lceil\log _{2} m\right\rceil$. This time is linear as a function of $n=r m$. Then $M$ interleaves $\vec{x}$ and $y^{k}$ on a separate track, and a single pass that checks for matches between \# signs marks all the occurrences of $y$ in $\vec{x}$ (if any).

(b) Suppose $m$ is even. $M$ first uses two passes to divide $\vec{x}$ into the "odd list" $x_{1} @^{r} x_{3} @^{r} \ldots x_{m-1} @^{r}$ and the "even list" $@^{r} x_{2} @^{r} x_{4} @^{r} \ldots @^{r} x_{m}$. Single passes then convert these to $x_{1} @^{3 r} x_{3} @^{3 r} \ldots x_{m-1} @^{3 r}$ and $@^{2 r} x_{2} @^{3 r} x_{4} @^{3 r} \ldots @^{3 r} x_{m}$. A pull step that writes the second over the first but translates @ to $B$ then produces $\vec{x}^{\prime}:=$ $x_{1} @^{r} x_{2} @^{r} x_{3} @^{r} \ldots @^{r} x_{m}$. If $m$ is odd then the "odd list" is $x_{1} @^{r} x_{3} @^{r} \ldots @^{r} x_{m}$ and the "even list" is $@^{r} x_{2} @^{r} x_{4} @^{r} \ldots @^{r} x_{m-1} @^{r}$, but the final result $\vec{x}^{\prime}$ is the same. By a similar process $M$ converts $\vec{y}$ to $\vec{y}^{\prime}:=@^{r} y_{1} @^{r} y_{2} \ldots @^{r} y_{m} @^{r}$. Writing $\vec{y}^{\prime}$ on top of $\vec{x}^{\prime}$ and translating @ to $B$ then yields shuffle $(\vec{x}, \vec{y})$. This requires only a constant number of passes.

A monoid is a set $H$ together with a binary operation o defined on $H$, such that $\circ$ is associative and $H$ has an element that is both a right and a left identity for $\circ$. We fix attention on the following representation of the monoid of transformations $\mathcal{M}_{S}$ of a finite-state machine $S . \mathcal{M}_{S}$ acts on the state set $Q$ of $S$ and is generated by the functions $\left\{g_{c}: c \in \Sigma\right\}$ defined by $g_{c}(q)=\delta(q, c)$ for all $q \in Q$, by letting $\circ$ be composition of maps on $Q$, and closing out the $g_{c}$ under $\circ$. Here we ignore 
the output function $\rho$ of $S$, intending to use it once the trajectory of states $S$ enters on an argument $z$ is computed. We also remark that $\mathcal{M}_{S}$ need not contain the identity mapping on $Q$, though it does no harm for us to adjoin it. By using known decomposition theorems for finite transducers [47, 32, 48], we could restrict attention to the cases where each $g_{c}$ either is the identity on $Q$ or identifies two states (a "reset machine") or each $g_{c}$ is a permutation of $Q$ and $\mathcal{M}_{S}$ is a group (a "permutation machine"; cf. [17]). These points do not matter here. We encode each state in $Q$ as a binary string of some fixed length $k$, and encode each element $g$ of $\mathcal{M}_{S}$ by the list $q \# g(q) \# \ldots$ over all $q \in Q$. Without loss of generality we extend $Q$ to $Q^{\prime}:=\left\{0, \ldots, 2^{k}-1\right\}$ and make $g$ the identity on elements $q \geq n$.

The word problem for monoids is: given a list $\vec{g}:=g_{n} g_{n-1} \cdots g_{2} g_{1}$ of elements of the monoid, not necessarily distinct, compute the representation of $g_{n} \circ g_{n-1} \circ \ldots \circ g_{2} \circ$ $g_{1}$. Let us call the following the trajectory problem: given $\vec{g}$ and some $w \in\{0,1\}^{k}$, compute the $n$-tuple $\left(g_{1}(w), g_{2}\left(g_{1}(w)\right), \ldots, \vec{g}(w)\right)$. The basic idea of the following is that "parallel prefix sum" is $\mu_{1}$-efficient on a BM.

Lemma 6.2. There is a fixed BM $M$ that, for any size parameter $k$, solves the word and trajectory problems for monoids acting on $\{0,1\}^{k}$ in $\mu_{1}$-time $O\left(n \cdot k 2^{k}\right)$. In particular, these problems for any fixed finite monoid belong to TLIN.

Proof. Let $T$ be a TM which, for any $k$, composes two mappings $h_{1}, h_{2}:\{0,1\}^{k} \rightarrow$ $\{0,1\}^{k}$ using the above representation. For ease of visualization, we make $T$ a singletape TM which on any input of the form $h_{2} \# h_{1} \#$ uses only the $2 k \cdot 2^{k}$ cells occupied by the input as workspace, and which outputs $h_{2} \circ h_{1} \#$ shuffled with '@' symbols so that the output has the same length as the input. We also program $T$ so that on input $h \#, T$ leaves $h$ unchanged. The running time $t(k)$ of $T$ depends only on $k$ and is $O\left(k 2^{k}\right)^{2}$. As in Example 2.4, we can create a GST $S$ whose input alphabet is the ID alphabet of $T$, such that for any nonhalting ID $I$ of $T, S(I)$ is the unique ID $J$ such that $I \vdash_{T} J$.

The BM $M$ operates as follows on input $\vec{g}:=g_{n} \# g_{n-1} \# \cdots \# g_{2} \# g_{1} \#$. It first saves $\vec{g}$ in cells $\left[\left(n k \cdot 2^{k}+1\right) \ldots\left(2 n k \cdot 2^{k}\right)\right]$ of a separate storage track. We may suppose that $n$ is even; if $n$ is odd, $g_{n}$ is left untouched by the current phase of the recursion. $M$ first sets up the initial ID of $T$ on successive pairs of maps, viz. $\wedge q_{0} g_{n} \# g_{n-1} \# \wedge q_{0} g_{n-2} \# g_{n-3} \# \cdots \wedge q_{0} g_{2} \# g_{1} \#$. Then $M$ invokes $S$ in repeated leftto-right pulls, until all simulated computations by $T$ have halted. Then $M$ erases all the @s, leaving $\left(g_{n} \circ g_{n-1}\right) \#\left(g_{n-2} \circ g_{n-3}\right) \# \cdots\left(g_{2} \circ g_{1}\right) \#$ on the tape. The number of sweeps is just $t(k)$, and hence the total $\mu_{1}$-time of this phase is $\leq 2 t(k) \cdot n=O(n)$.

$M$ copies this output to cells $\left[\left((n / 2) k \cdot 2^{k}+1\right) \ldots\left(n k \cdot 2^{k}\right)\right]$ of the storage track, and then repeats the process, until the last phase leaves $h:=g_{n} \circ g_{n-1} \circ \ldots \circ g_{2} \circ g_{1}$ on the tape. Since the length of the input halves after each phase, the total $\mu_{1}$-time is still $O(n)$. This finishes the word problem.

To solve the trajectory problem, $M$ uses the stored intermediate results to recover the path $\left(w, g_{1}(w), g_{2}\left(g_{1}(w)\right), \ldots, h(w)\right)=:\left(w, w_{1}, w_{2}, \ldots, w_{n}\right)$ of the given $w \in\{0,1\}^{k}$. Arguing inductively from the base case $(w, h(w))$, we may suppose that $M$ has just finished computing the path $\left(w, w_{2}, w_{4}, \ldots, w_{n-2}, w_{n}\right) . M$ shuffles this with the string $g_{1} \# g_{3} \# g_{5} \# \ldots \# g_{n-1}$ and then simulates in the above manner a TM $T^{\prime}$ that given a $g$ and a $w$ computes $g(w)$. All this takes $\mu_{1}$-time $O(n)$.

The following presupposes that all BMs $M$ are described in such a way that the alphabet $\Gamma_{M}$ of $M$ can be represented by a uniform code over $\{0,1\}^{*}$. This code is extended to represent monoids $\mathcal{M}$ as described above. 
THEOREM 6.3. There is a $B M M_{U}$ and a computable function code such that for any $B M M$ and rational $d \geq 1$, there is a constant $K$ such that for all inputs $x$ to $M$, $M_{U}$ on input $(\operatorname{code}(M)$, code $(x), d)$ simulates $M(x)$ within $\mu_{d}$-time $K \cdot \mu_{d}$-time $(M, x)$.

Proof. $M_{U}$ uses the alphabet $\Gamma_{U}:=\{0,1, @, \$,(0, \#),(1, \#),(@, \#), \Delta, B\}$. By Theorem 2.12, we may suppose that $M$ has a single GST $S=\left(Q, \Gamma_{M}, \Gamma_{M}, \delta, \rho, s_{0}\right)$. Let $k:=\left\lceil\log _{2}\left|\Gamma_{M}\right|\right\rceil$, and let $l$ be the least integer above $\log _{2}|Q|$ that is a multiple of $k$. The code function on strings codes each $c \in \Gamma_{M}$ by a $0-1$ string of length $k$, except that the last bit of code $(c)$ is combined with \# and $B$ is coded by ${ }^{k-1}$ (@, \#).

The monoid $\mathcal{M}$ of transformations of $S$ is encoded by a $k$-tuple of elements of the form code $(c) \operatorname{code}\left(g_{c}\right)$ over all $c \in \Gamma_{M}$. Here $\operatorname{code}\left(g_{c}\right)$ is as described before Lemma 6.2. Dummy states are added to $Q$ so that $\operatorname{code}\left(g_{c}\right)$ has length exactly $2 l \cdot 2^{l}$; then $\operatorname{code}(\mathcal{M})$ has length exactly $2^{k}\left(k+2 l \cdot 2^{l}\right)$. Let $C$ be the maximum number of symbols written in any transition of $M$. The code of $S$ includes a string code $(\rho)$ that gives the output for each transition in $\delta$, padded out with @ symbols to length exactly $C$ (i.e., length $C k$ under code). The rest of the code of $M$ lists the mode-change information for each terminal state of $S$. Finally, the input $x$ to $M$ is represented by the string $\operatorname{code}(x)$ of length $|x| 2^{k}$.

$M_{U}$ has four tracks: one for the main tape of $M$, one for the code of $M$, one for simulating passes by $M$, and one for scratchwork. $M_{U}$ uses $d$ to compute $e:=$ $\mu_{d}(a)$, and follows just the part of the proof of Theorem 4.15 that locates the cells $a_{j}:=a \pm 2^{j-1} e$, in order to drop $\$$ characters there. This allows $M_{U}$ to pull off from its main track in cells $\left[a \ldots a_{j}\right]$ the code of the first $m:=2^{j-1} e / 4 k$ characters of the string $x$ that $M$ reads in the pass being simulated. (If this pass is a put rather than a pull, then $e=1$ and $x$ is in cells $\left[1 \ldots 2^{j-1}\right]$.) Then $M_{U}$ changes code $(z)$ to

$$
z^{\prime}:=\left(\operatorname{code}\left(z_{0}\right)\right)^{j} \cdot\left(\operatorname{code}\left(z_{1}\right)\right)^{j} \cdots\left(\operatorname{code}\left(z_{m-1}\right)\right)^{j},
$$

where $m:=|z|$ and $j:=2^{k}\left(1+2(l / k) 2^{l}\right)$. This can be done in linear $\mu_{1}$-time by iterating the procedure for shuffle in Lemma 6.1(b). Now for each $i, 0 \leq i \leq m-1$, the $i$ th segment of $z^{\prime}$ has the same length as code $(\mathcal{M})$. Next, $M$ uses "recursive doubling" to change $\operatorname{code}(\mathcal{M})$ to $(\operatorname{code}(\mathcal{M}))^{m}$. This also takes only $O(m)$ time. Then the strings $z^{\prime}$ and $(\operatorname{code}(\mathcal{M}))^{m}$ are interlaced on the scratchwork track. A single pass that matches the labels code $(c)$ to segments of $z^{\prime}$ then pulls out the word $g_{z}:=g_{z_{0}} \cdot g_{z_{1}} \cdots g_{z_{m-1}}$.

$M$ evaluates this word by the procedure of Lemma 6.2, yielding the encoded trajectory $s^{\prime}:=\left(s_{0}, s_{1}, \ldots, s_{m}\right)$ of $S$ on input $z$. By a process similar to that of the last paragraph, $M_{U}$ then aligns $s^{\prime}$ with $(\operatorname{code}(\rho))^{m}$ and interleaves them, so that a single pass pulls out the output $y$ of the trajectory. Then $\operatorname{code}(y)$ is written to the main tape, erasing the symbols $\Delta$ used for padding and translating @ to $B$. The terminal state $s_{m}$ of the trajectory is matched against the list that gives the mode information for the next pass of $M$ (Lemma 6.1a), and $M_{U}$ changes its mode and/or current address accordingly.

If the original pass by $M$ cost $\mu$-time $\mu(a)+m+\mu(b)$, then the simulation takes $\mu$ time $\mu(4 a)+O(m)+\mu(4 b)$. The constant in the ' $O(m)$ ' depends only on $M$. We have described $M_{U}$ as though there were no validity restrictions on passes, but Theorems 4.1 and 2.12 convert $M_{U}$ to a basic BM while keeping the constant overhead on $\mu_{d}$-time.

Remarks: This result implies that there is a fixed, finite collection of GSTs that form an efficient "universal chipset." It might be interesting to explore this set in greater detail. The constant on the ' $O(m)$ ' is on the order of $2^{2(l+k)}(l+k)$. We 
inquire whether there are other representations of finite automata or their monoids that yield notably more efficient off-line simulations than the standard one used here. The universal simulation in Theorem 6.3 does not preserve $w$ or $\mu_{d^{-}}$acc individually because it uses the method of Theorem 4.15 to compensate for its lack of "foreknowledge" about where a given block move by $M$ will exit. The simulation does preserve memory-efficiency, on account of Proposition 5.4. If, however, we suppose that $M$ is already self-delimiting in a way made transparent by code, then we obtain constant overheads in both $w$ and $\mu$-acc, and the simulation itself becomes independent of $\mu$.

THEOREM 6.4. There is a BM $M_{U}$ and a computable function code such that for any memory cost function $\mu$ and any self-delimiting $B M M$, there is a constant $K$ such that for all inputs $x$ to $M, M_{U}$ on input $x^{\prime}=(\operatorname{code}(M)$, code $(x))$ simulates $M(x)$ with $w\left(U, x^{\prime}\right) \leq K w(M, x)$ and $\mu-a c c\left(U, x^{\prime}\right) \leq K \mu-a c c(M, x)$.

Proof. The function code is changed so that it encodes the endmarkers of $M$ by strings that begin with ' $\$$ '. Then $M_{U}$ pulls off the portion $x$ of its main track up to $\$$. The rest of the operation of $M_{U}$ is the same, and the bounds now require only the tracking property of $\mu$. (If the notion of "self-delimiting" is weakened as discussed before Definition 3.3, then we can have $M_{U}$ first test whether a GST $S$ exits on the second symbol of $x$.) प

To use these results for diagonalization, we need two preliminary lemmas. Recall that a function $t$ is $\mu$-time constructible if $t(n)$ is computable in binary notation in $\mu$-time $O(t(n))$. Since all of $n$ must be read, $t$ must be $\Omega(\log n)$.

Lemma 6.5. If a BM $M$ is started on an input of length $n$, then any pass by $M$ either takes $\mu_{1}$-time $O(n)$, or else no more than doubles the accumulated $\mu$-time before the pass.

Proof. Any portion of the tape other than the input that is read in the pass must have been previously written in some other pass or passes. (Technically, this uses our stipulation that $B$ is an endmarker for GSTs.) Thus the conclusion follows.

Lemma 6.6. For any memory cost function $\mu$ that is $\mu$-time constructible, a $B M M$ can maintain a running total of its own $\mu$-time with only a constant-factor slowdown.

Proof. To count the number $m=|b-a|+1$ of transitions made by one of its GST chips $S$ in a given pass, a BM $M$ can invoke a "dummy copy" of $S$ that copies the content $x$ of the cells up to where $S$ exits to a fresh track, and then count $|x|$ on that track by the $O(m)$-time procedure of Example 2.3. Then $M$ invokes $S$ itself and continues operation as normal. Since $\mu$ is $\Omega(\log n)$, the current address $a$ can be copied and updated on a separate track in $\mu$-time $O(\mu(a))$. Also in a single pass, $M$ can add $a$ and $m$ in $\mu$-time $O(\mu(a)+m)$, and thus obtain $b$ itself. $M$ then calculates $\mu(b)$ in $\mu$-time $O(\mu(b))$, and finally adds $k:=\mu(a)+m+\mu(b)$ to its running total $t$ of $\mu$-time. In case $t$ is much longer than $k$, we want the work to be proportional to $|k|$, not to $|t|$. Standard "carry-save" techniques, or alternatively an argument that long carries cannot occur too often, suffice for this. $\square$

TheOREM 6.7. Let $d \geq 1$ be rational, and let $t_{1}$ and $t_{2}$ be functions such that $t_{2}$ is $\mu_{d}$-time constructible, and $t_{1}$ is o $\left(t_{2}\right)$. Then $\mathrm{D} \mu \mathrm{TIME}\left[t_{1}\right]$ is properly contained in $\mathrm{D} \mu \mathrm{TIME}\left[t_{2}\right]$.

Proof. The proof of Theorem 6.3 encoded BMs $M$ over the alphabet $\Gamma_{U}$, but let code ${ }^{\prime}$ re-code $M$ over $(00 \cup 11)^{*}$. We build a $\mathrm{BM} M_{D}$ that accepts a language 
$D \in \mathrm{D} \mu \mathrm{TIME}\left[t_{2}\right] \backslash \mathrm{D} \mu \mathrm{TIME}\left[t_{1}\right]$ as follows. $M_{D}$ has two extra tracks on which it logs its own $\mu$-time, as in Lemma 6.6. On any input $x, M_{D}$ first calculates $n:=|x|$, and then calculates $t_{2}(n)$ on its "clock track." Next, $M_{D}$ lets $w$ be the maximal initial segment of doubled bits of $x$. Since the set $\{\operatorname{code}(M): M$ is a BM $\}$ is recursive, $M_{D}$ can decide whether $w$ is the code $e^{\prime}$ of a BM $M$ in some time $U(n)$. The device of using $w$ ensures that there are $\infty$-many inputs in which any given $\mathrm{BM} M$ is presented to $M_{D}$. If $w$ is not a valid code, $M_{D}$ halts and rejects.

If so, $M_{D}$ runs $M_{U}$ on input code $(M) \cdot \operatorname{code}(x)$, except that after every pass by $M_{U}, M_{D}$ calculates the $\mu$-time of the pass and subtracts it from the total on its clock tape. If the total ever falls below $t_{2}(n) / 2, M_{D}$ halts and rejects. Otherwise, if the simulation of $M(x)$ finishes before the clock "rings," $M_{D}$ rejects if $M$ accepts, and accepts if $M$ rejects. By Lemma 6.5, the total $\mu$-time of $M_{D}$ never exceeds $t_{2}(n)$.

Now let $L$ be accepted by a BM $M_{L}$ that runs in $\mu$-time $t_{1}(n)$. Let $K_{1}$ be the constant overhead for $M_{U}$ to simulate $M_{L}$ in Theorem 6.3 , and let $K_{2}$ be the overhead in Lemma 6.6. Since $t_{1}$ is $o\left(t_{2}\right)$, there exists an $x$ such that $t_{2}(|x|) / t_{1}(|x|)>4 K_{1} K_{2}$, the maximal initial segment $w \in(00 \cup 11)^{*}$ of $x$ is $\operatorname{code}^{\prime}\left(M_{L}\right)$, and $U(|w|)<|x|$. Then the simulation of $M_{L}(x)$ by $M_{D}$ finishes within $\mu$-time $(1 / 2) t_{2}(|x|)$, and $M_{D}(x) \neq$ $M_{L}(x)$.

It is natural to ask whether the classes $\mathrm{D} \mu_{d} \operatorname{TIME}[t(n)]$ also form a tight hierarchy when $t$ is held constant and $d$ varies. The next section relates this to questions of determinism versus nondeterminism.

We observe finally that the BM in its original, reduced, and buffer forms all give the same definition of $\mathrm{D} \mu_{\log } \operatorname{TIME}[t(n)]$, and we have:

THEOREM 6.8. For any time functions $t_{1}, t_{2}$ such that $t_{1}(n) \geq n, t_{1}=o\left(t_{2}\right)$, and $t_{2}$ is $\mu_{\log }$-time constructible, $\mathrm{D} \mu_{\log }$ TIME $\left[t_{1}\right]$ is properly contained in $\mathrm{D} \mu_{\log } \operatorname{TIME}\left[t_{2}\right]$.

Proof. Here the strict boundary condition is not an issue, but the efficient universal simulation still requires delimiting the read block in advance. The idea is to locate cells $a_{1}, a_{2}, a_{3} \ldots$ in the proof of Theorem 4.15 without addressing by the following trick. As in Theorem 4.16, the current address $a_{0}$ is already stored and $e=\left|a_{0}\right|$. In a rightward pull, rather than add $a_{0}+e, M^{\prime}$ puts $a_{0}$ itself in binary rightward from cell $a_{0}$ on a separate track, appending an endmarker $\$$. By "recursively doubling" the string $a_{0}, M^{\prime}$ can likewise delimit the cells $a_{2}, a_{3}, \ldots$ Leftward pull steps are handled similarly, and put steps do not need $\mu_{\log }\left(a_{0}\right)$ at all. This is all that is needed for the efficient universal simulation. The remainder follows as above, since $\mu_{\log }$ is $\mu_{\log }$-time constructible - in fact, $\mu_{\log }(a)=|a|$ is computable in $\mu_{1}$-time $O(|a|) . \square$

A similar statement holds for the perhaps-larger $\mu_{\log }$-time classes for the BM variants that do use addressing.

7. Complexity Theory and the BM Model. Our first result shows that the construction in the Hennie-Stearns theorem [33], which states that any multitape TM that runs in time $t(n)$ can be simulated by a 2-tape TM in time $t(n) \log t(n)$, is memory-efficient on the $\mathrm{BM}$ under $\mu_{1}$. It has been observed in general that this construction is an efficient caching strategy. DTIME $[t(n)]$ refers to TM time, and DLIN stands for DTIME $[O(n)]$.

Theorem 7.1. For any function $t, \operatorname{DTIME}[t(n)] \subseteq \mathrm{D} \mu_{1} \operatorname{TIME}[t(n) \log t(n)]$.

Proof. With reference to the treatment in [36], let $M_{1}$ be a multitape TM with alphabet $\Gamma$ that runs in time $t(n)$, and let $M_{2}$ be the two-tape TM in the proof. 
The $k$-many tapes of $M_{1}$ are simulated on $2 k$-many tracks of the first tape of $M_{2}$ so that all tape heads of $M_{1}$ are maintained on cell 0 of each track. $M_{2}$ uses its second tape only to transport blocks of the form $\left[2^{j-1} \ldots 2^{j}-1\right]$ from one part of the first tape to another. The functions used in these moves are homomorphisms between the alphabets $\Gamma^{2 k}$ and $\Gamma^{k}$ that pack and unpack characters in blocks. Thus a BM $M_{3}$ simulating $M_{2}$ can compute each move in a single GST pass. By the structure of the blocks, any pass that incurs a memory-access charge of $\mu_{1}\left(2^{j}\right)=2^{j}$ simulates at least $2^{j-1}$ moves of $M_{2}$. Hence the work and the $\mu_{1}$ charges to $M_{3}$ are both $O(t(n) \log t(n)) . \square$

We do not know whether the random access capability of a BM can be exploited to give an $O(t \log t)$ simulation that holds the work to $O(t)$, even for $\mu=\mu_{\log }$. Indeed, $O(t \log t)$ is the best bound we know for all memory cost functions $\mu$ between $\mu_{\log }$ and $\mu_{1}$. One consequence of this proposition is that sets in DLIN can be padded out to sets in TLIN.

COROLlary 7.2 .

(a) For every $L \in \mathrm{DLIN}$, the language $\left\{x \# 0^{|x| \log |x|}: x \in L\right\}$ belongs to TLIN.

(b) TLIN contains $\mathrm{P}$-complete languages, so TLIN $\subseteq \mathrm{NC} \Longleftrightarrow \mathrm{P}=\mathrm{NC}$.

Hence it is unlikely that all TLIN functions can be computed in polylog-many passes like the examples in this paper. If a BM quickly compresses the amount of information remaining to be processed into cells $[0 \ldots \sqrt{n}]$, it can then spend $O(\sqrt{n})$ time accessing these cells in any order desired and still run in linear $\mu_{1}$-time.

THEOREM 7.3. Let $M$ be a BM that runs in $\mu$-time $t(n)$ and space $s(n)$. Then we can find a DTM T that simulates $M$ in time $O[t(n) s(n) / \mu(s(n))]$.

Proof. $T$ has two tapes: one for the main tape of $M$, and one used as temporary storage for the output in passes. (If $M$ has the buffer mechanism, then the second tape of $T$ simulates the buffer.) Let $s$ stand for $s(n)$. Consider a move by $M$ that changes the current address $a$ to $\lfloor a / 2\rfloor$. $T$ can find this cell in at most $3 a / 2$ steps by keeping count with its second tape. Since $s / a \geq 1$, the tracking property $\mu(N a) \leq N \mu(a)$ with $N:=s / a$ gives $a / \mu(a) \leq s / \mu(s)$. Hence the ratio of the time used by $T$ to the $\mu$-time charged to $M$ stays $O[s / \mu(s)]$. The same holds for the moves $a:=2 a$ and $a:=2 a+1$. $T$ has every GST $S$ of $M$ in its finite control, and simulates a pull by writing $S[a \ldots b]$ to its second tape, moving to cell 0, copying $S[a \ldots b]$ over the first tape, and moving back to cell $a$. Both this and the analogous simulation of a put by $T$ take time $O(a+b)$, and even the ratio of this to the memory access charges $\mu(a)+\mu(b)$, not even counting the number of bits processed by $M$, keeps the running total of the time logged by $T$ below $t(n) s / \mu(s)$.

Corollary 7.4. For any time bound $t(n) \geq n$, D $\mu_{1} \operatorname{TIME}[t(n)] \subseteq \operatorname{DTIME}[t(n)]$. In particular, TLIN $\subseteq$ DLIN. $\square$

More generally, for any $d \geq 1, \mathrm{D} \mu_{d} \operatorname{TIME}[t(n)] \subseteq \operatorname{DTIME}\left[t^{2-(1 / d)}(n)\right]$. Allowing TMs to have $d$-dimensional tapes brings this back to a linear-time simulation:

LEMMA 7.5. For any integer $d \geq 1$ and time bound $t(n) \geq n$, a $B M M$ that runs in $\mu_{d}$-time $t(n)$ can be simulated in time $O(t(n))$ by a d-dimensional TMT.

Proof. $T$ has one $d$-dimensional tape on which it winds the main tape of $M$ in a spiral about the origin, and one linear tape on which it buffers outputs by the GST $S$ of $M$. In any pass that incurs a $\mu_{d}$ charge of $a^{1 / d}, T$ can walk between cell $a$ and the origin within $a^{1 / d}$ steps and complete the move. $\square$ 
Let us say that a language or function in $\mathrm{D} \mu_{d} \operatorname{TIME}[O(n)]$ has dimension d. For a problem above linear time, we could say that its dimensionality is the least $d$, if any, for which the problem has relatively optimal BM programs that are $\mu_{d}$-efficient (see Definition 2.10). The main robustness theorem is our justification for this concept of dimensionality. Lemma 7.5 says that it is no less restrictive than the older concept given by $d$-dimensional Turing machines. For $d>1$ we suspect that it is noticeably more restrictive. The $d$-dimensional tape reduction theorem of Paul, Seiferas, and Simon [58] gives $t^{\prime}(n)$ roughly equal to $t(n)^{1+1 / d}$, and when ported to a BM, incurs memory access charges close to $t(n)^{1+2 / d}$. Intuitively, the problem is that a $d$-dimensional TM can change the direction of motion of its tape head(s) at any step, whereas this would be considered a break in pipelining for the simulating BM, and thus subject to a memory-access charge.

We write RAM-TIME ${ }^{\log }$ for time on the log-cost RAM. A log-cost RAM can be simulated with constant-factor overhead by a TM with one binary tree-structured tape and one standard worktape [57], and the latter is simulated in real time by a RAM-TM.

Proposition 7.6. For any time function $t$,

(a) RAM-TIME ${ }^{\log }[t(n)] \subseteq \mathrm{D} \mu_{\log } \operatorname{TIME}[t(n) \log t(n)]$.

(b) $\mathrm{D} \mu_{\log } \mathrm{TIME}[t(n)] \subseteq \mathrm{RAM}^{-T_{I M E}}{ }^{\log }[t(n) \log t(n)]$.

Proof. Straightforward simulations give these bounds. (The extra $\log t(n)$ factor in (b) dominates a factor of $\log \log n$ that was observed by [44] for the simulation of a TM (or RAM-TM) by a log-cost RAM.) $\square$

For quasilinear time, i.e. time qlin $=n(\log n)^{O(1)}$, the extra $\log n$ factors in Theorem 7.1 and Proposition 7.6 do not matter. Following Schnorr [65], we write DQL and NQL for the TM time classes DTIME $[$ qlin $]$ and NTIME[ qlin]. Gurevich and Shelah proved that RAM-TIME ${ }^{\log }[$ qlin $]$ is the same as deterministic nearly linear time on the RAM-TM and several other RAM-like models, and perhaps more surprisingly, that the nondeterministic counterparts of these classes are all equal to NQL.

COROLlary 7.7.

(a) $\mathrm{D} \mu_{1} \mathrm{TIME}[$ qlin $]=\mathrm{DQL}$.

(b) $\mathrm{D} \mu_{\log }$ TIME $[$ qlin $]=$ RAM-TIME ${ }^{\log }[$ qlin $] \subseteq$ NQL. $\square$

Hence the objective of separating the classes $\mathrm{D} \mu \mathrm{TIME}[O(n)]$, as $\mu$ varies from $\mu_{1}$ through $\mu_{d}$ to $\mu_{\log }$, by anything more than factors of $O(\log n)$, runs into the problem of whether $\mathrm{DQL} \neq \mathrm{NQL}$, which seems as hard as showing $\mathrm{P} \neq \mathrm{NP}$. Whether they can be separated by even one $\log n$ factor is discussed in the next section.

8. Open Problems and Further Research. The following languages have been much studied in connection with linear-time algorithms and nonlinear lower bounds. We suppose that the lists in $L_{d u p}$ and $L_{\text {int }}$ are all normal.

(a) Pattern matching: $L_{p a t}=\left\{p \# t:\left(\exists u, v \in\{0,1\}^{*}\right) t=u p v\right\}$.

(b) Element (non)distinctness: $L_{\text {dup }}=\left\{x_{1} \# \ldots \# x_{m}:(\exists i, j) i<j \wedge x_{i}=x_{j}\right\}$.

(c) List intersection: $L_{\text {int }}=\left\{x_{1} \# \ldots \# x_{m}, y_{1} \# \ldots \# y_{m}:(\exists i, j) x_{i}=y_{j}\right\}$.

(d) Triangle: $L_{\Delta}=\{A: A$ is the adjacency matrix of an undirected graph that contains a triangle $\}$.

$L_{\text {pat }}$ belongs to DLIN (see $[25,23]$ ), and was recently shown not to be solvable by a one-way non-sensing multihead DFA [42]. $L_{d u p}$ and $L_{i n t}$ can be solved in linear time 
by a RAM or RAM-TM that treats list elements as cell addresses. $L_{\Delta}$ is not believed to be solvable in linear time on a RAM at all. The best method known involves computing $A^{2}+A$, and squaring $n \times n$ integer matrices takes time approximately $N^{1.188}$, where $N=n^{2}$, by the methods of [19]. (For directed triangles, cubing $A$ is the best way known.)

Open Problem 1. Do any of the above languages belong to TLIN? If not, prove nonlinear lower bounds.

A BM can be made nondeterministic (NBM) by letting $\delta(q, c)$ be multiply valued, and more strongly, by using nondeterministic GSTs or GSM mappings in block moves. Define NTLIN to be linear time for NBMs of the weaker kind. Then all four of the above languages belong to NTLIN. Moreover, they require only $O(\log n)$ bits of nondeterminism.

Open Problem 2. Is NTLIN $\neq$ TLIN? For reasonable $\mu$ and time bounds $t$, is there a general separation of $\mathrm{N} \mu \mathrm{TIME}[t(n)]$ from $\mathrm{D} \mu \mathrm{TIME}[t(n)]$ ?

Grandjean $[27,28]$ shows that a few NP-complete languages are also hard for NLIN under TM linear time reductions, and hence by the theorem of [56] lie outside DLIN, not to mention TLIN. However, these languages seem not to belong to NTLIN, nor even to linear time for NBMs of the stronger kind. The main robustness theorem and subsequent simulations hold for the weaker kind of nondeterminism, but our proofs do not work for the stronger because they re-run the GST $S$ used in a pass. We suspect that different proofs will give similar results. A separation of the two kinds can be shown with regard to the pass count measure $R(n)$, which serves as a measure of parallel time (e.g. $R(n)=\operatorname{polylog}(n)$ and polynomial work $w(n)$ by deterministic BMs characterizes NC [62]). P. van Emde Boas [personal communication, 1994] has observed that while deterministic BMs and NBMs of the weaker kind belong to the second machine class of [68] with $R(n)$ as time measure, NBMs of the stronger kind have properties shown there to place models beyond the second machine class. Related to Problem 2 is whether the classes $\mathrm{D} \mu_{d} \operatorname{TIME}[O(n)]$ differ as $d$ varies. It is also natural to study memory-efficient reductions among problems.

The following idea for obtaining such separations and proving nonlinear lower bounds in $\mu$-time on a deterministic BM $M$ suggests itself: Let $\Gamma_{M, x}$ stand for the set of access points used in the computation of the BM $M$ on input $x$. In order for $M$ to run in linear $\mu$-time, $\Gamma_{M, x}$ must thin out at the high end of memory. In particular for $\mu=\mu_{1}$, there are long segments between access points that can be visited only a constant number of times. The technical difficulty is that block moves can still transport information processed in low memory to these segments, and the proof of Theorem 7.1 suggests that a lower bound of $\Omega[n \log n]$ may be the best achievable in this manner. In general, we advance the BM as a logical next step in the longstanding program of proving nonlinear lower bounds for natural models of computation. In particular, we ask whether the techniques used by Dietzfelbinger, Maass, and Schnitger [20] to obtain lower bounds for Boolean matrix transpose and several sorting-related functions on a certain restricted two-tape TM can be applied to the differently-restricted kind of two-tape TM in Theorems 7.1 and 7.3. The latter kind is equivalent to a TM with one worktape and one pushdown store with the restriction that after any POP, the entire store must be emptied before the next Push.

We have found two variants to the BM model that seem to depart from the cluster of robustness results shown in this paper. They relate to generally-known issues of 
delay in computations. The first definition is the special case for GSTs of Manacher's notion of a "fractional on-line RAM algorithm with steady-paced output" [53].

Definition 8.1. Let $d \geq 0$ and $e \geq 1$ be integers. A GST $S$ runs in fixed output delay $d / e$ if for every terminal trajectory $\left(q_{0}, x_{0}, q_{1}, \ldots, x_{m-1}, q_{m}\right)$, and each $i \leq m-2,\left|\rho\left(q_{i}, x_{i}\right)\right|=d$ if e divides $i+1,=0$ otherwise. For the exiting transition, $\left|\rho\left(q_{m-1}, x_{m-1}\right)\right|$ depends only on $(m \bmod e)$. The quantity $C:=d / e$ is called the expansion factor of $S$.

Note that the case $d=0$ is allowed. Every GST function $g$ can be written as $e \circ f$, where $f$ is fixed-delay and $e$ is an erasing homomorphism: pad each output of the GST for $g$ to the same length with '@' symbols, and let $e$ erase them. A $k$-input GST with stationary moves allowed may keep any of its input heads stationary in a transition. Such a machine can be converted to an equivalent form coded like an ordinary GST in which every state $q$ has a label $j \in\{1, \ldots, k\}$ such that $q$ reads and advances only the head on tape $j$.

DEFINITION 8.2.

(a) A BM runs in fixed output delay if every GST chip in $M$ runs in fixed output delay.

(b) A pause buffer BM is a BM with buffer whose put steps may use 2-input GSTs with variable input delay (cf. Proposition 4.3).

Put another way, the BM model presented in this paper requires fixed delay in reading input but not in writing output, while (a) requires both and (b) requires neither. We did not adopt (b) because we feel that stationary moves by a 2-GST in the course of a pass require communication between the heads, insofar as further movements depend on the current input symbols, and hence should incur memory-access charges. We defend our choice against a similar criticism that would require (a) by contending that in a single-tape GST pass, the motion of the read head is not affected by the write head, and the motion of the write head depends only on local factors as bits come in to it. Also, every BM has a limit $C$ on the number of output bits per input bit read by a GST. The main robustness theorem, in particular the ability to forecast the length of the output of a pass by fixed-delay means shown in Theorem 4.15, satisfy our doubts about this.

The robustness results in this paper do carry over to the case of fixed output delay:

TheOREM 8.3. For any rational $d \geq 1$, the fixed-delay restrictions of the BM and all the variants defined in Section 3 simulate each other up to constant factors in $\mu_{d}$-time.

Proof. All auxiliary operations in the simulations in Section 4 use GSTs that run in fixed output delay, except for the second, unpadded run of the GST $S$ in Theorem 4.15. However, if $S$ already runs in fixed output delay, so does this run. $\square$

Under the proof of Theorem 2.12 the corresponding notion for the reduced form of the model is "fixed delay after the initial transition." Our proof of efficient universal simulation does not quite carry over for fixed output delay because the quantities $k$ and $l$ in the proof of theorem 6.3 may differ for different $M$. The operations that pull off the word $g_{z}$ and the padded output code $(y)$ run in "stride" a function of $k$ and $l$, but this is not fixed. We believe that the proof can be modified to do so under a different representation scheme for monoids. 
Whether a similar robustness theorem holds for the pause-buffer BM leads to an open problem of independent interest: can every $k$-input GST be simulated by a composition tree of 2-input GSTs when stationary moves are allowed? The questions of the power of both variants versus the basic BM can be put in concrete terms.

Open Problem 3. Can the homomorphism $E r_{2}:\{0,1,2\}^{*} \rightarrow\{0,1\}^{*}$, which erases all 2's in its argument, be computed in linear $\mu_{1}$-time by a BM that runs in fixed output delay?

Open Problem 4. For every 2-input GST $S$ with stationary moves allowed, does the function $S^{\prime}(x \# y):=S(x, y)$ belong to TLIN?

THEOREM 8.4.

(a) The answer to Problem 3 is 'yes' iff for every memory cost function $\mu$ and $B M M$, there is a $B M M^{\prime}$ that runs in fixed output delay and simulates $M$ linearly under $\mu$.

(b) The answer to Problem 4 is 'yes' iff for every memory cost function $\mu$ and pause-buffer $B M M$, there is a BM $M^{\prime}$ that simulates $M$ linearly under $\mu$.

Proof. For the forward implication of (a), $M^{\prime}$ pads every output by $M$ with @ symbols, coding the rest over $\{0,1\}^{*}$, and runs $E r @$ on a separate track to remove the padding. That of (b) is proved along the lines of Proposition 4.3. The reverse implications are immediate, and all this needs only the tracking property of $\mu$. .

Alon and Maass [4] prove substantial time-space tradeoffs for the related "sequence equality" problem $\mathrm{SE}[n]$ : given $x, y \in\{0,1,2\}^{n}$, does $\operatorname{Er}_{2}(x)=E r_{2}(y)$ ? We inquire whether their techniques, or those of [54], can be adapted to the BM. The BM in Theorem 7.1 runs in output delay $1 / 2,1$, or 2 for all passes, so the two kinds of BM can be separated by no more than a log factor. A related question is whether every language in TLIN, with or without fixed output delay, has linear-sized circuits.

Further avenues for research include analyzing implementations of certain important algorithms on the BM, as done for the $\mathrm{BT}$ and $\mathrm{UMH}$ in $[2,5]$. Here the $\mathrm{BM}$ is helped by its proximity to the Pratt-Stockmeyer vector machine, since conserving memory-access charges and parallel time often lead to similar methods. One can also study storage that is expressly laid out on a $2 \mathrm{D}$ grid or in $3 \mathrm{D}$ space, where a pass might be defined to follow either a 1D line or a 2D plane. We expect the former not to be much different from the BM model with its 1D tape, and we also note that CDROM and several current 2D drive technologies use long 1D tracks. The important issue may not be so much the topology of the memory itself, but whether "locality is one-dimensional" for purposes of pipelining.

Last, we ask about meaningful technical improvements to the simulations in this paper. The lone obstacle to extending the main robustness theorem for $\mu=\mu_{\log }$ is the simulation of random access by tree access in Lemma 4.7. The constants on our universal simulation are fairly large, and we seek a more-efficient way of representing monoids and computing the products. Two more questions are whether the BM loses power if the move option $a:=2 a+1$ is eliminated, and whether the number $m$ of markers in a finger BM can be reduced to $m-1$ or to 4 without multiplying the number of block moves by a factor of $\log t(n)$.

9. Conclusion. In common with motivations expressed in [2] and [5], the BM model fosters a finer analysis of many theoretical algorithms in terms of how they use memory, and how they really behave in running time when certain practicalities 
of implementation are taken into account. We have shown that the BM model is quite robust, and that the concept of functions and languages being computable in a memory-efficient manner does not depend on technical details of setting up the model. The richer forms of the model are fairly natural to program, providing random access and the convenience of regarding finite transductions such as addition and vector Booleans as basic operations. The tightest form of the model is syntactically simple, retains the bit-string concreteness of the TM, and seems to be a tractable object of study for lower bound arguments. The robustness is evidence that our abstraction is "right."

In contrast to the extensive study of polynomial-time computation, very little is known about linear time computation. Owing to an apparent lack of linear-time robustness among various kinds of TMs, RAMs, and other machines, several authorities have queried their suitability as a model for computation in $O(n)$ time. Since we have $\mu$ as a parameter we have admittedly not given a single answer to the question "What is Linear Time?", and leave TLIN, D $\mu_{2} \operatorname{TIME}[O(n)]$, and $\mathrm{D} \mu_{3} \operatorname{TIME}[O(n)]$ as leading candidates. However, the BM model does supply a robust yardstick for assessing the complexity of many natural combinatorial problems, and for investigating the structure of several other linear-time complexity classes. It has a tight deterministic time hierarchy right down to linear time. The efficient universal simulator which we have constructed to show this result uses the word problem for finite monoids in an interesting manner. The longstanding program of showing nonlinear lower bounds in reasonable models of computation has progressed up to machines apparently just below the BM (under $\mu_{1}$ ) in power, so that attacking the problems given here seems a logical next step. The authors of [3] refer to the "challenging open problem" of extending their results when bit-manipulations for dissecting records are available. The bit operations given to the BM seem to be an appropriate setting for this problem. A true measure of the usefulness of the BM model will be whether it provides good ground for developing and connecting methods that solve older problems not framed with the term "BM." We offer the technical content of this paper as appropriately diligent spadework.

Acknowledgments.. I would like to thank Professor Michael C. Loui for comments on preliminary drafts of this work, and for bringing [2] and [5] to my attention. Professors Pierre McKenzie and Gilles Brassard gave me a helpful early opportunity to test these ideas at an open forum in a University of Montreal seminar. Special thanks are due to Professors Klaus Ambos-Spies, Steven Homer, Uwe Schöning, and other organizers of the 1992 Schloss Dagstuhl Workshop on Structural Complexity for inviting me to give a presentation out of which the present work grew. Last, I thank an anonymous referee and several colleagues for helpful suggestions on nomenclature and presentation.

\section{REFERENCES}

[1] A. Aggarwal, B. Alpern, A. Chandra, and M. Snir, A model for hierarchical memory, in Proc. 19th Annual ACM Symposium on the Theory of Computing, 1987, pp. 305-314.

[2] A. Aggarwal, A. Chandra, And M. Snir, Hierarchical memory with block transfer, in Proc. 28th Annual IEEE Symposium on Foundations of Computer Science, 1987, pp. 204-216.

[3] A. Aggarwal and J. Vitter, The input-output complexity of sorting and related problems, Comm. Assn. Comp. Mach., 31 (1988), pp. 1116-1127.

[4] N. Alon And W. MaAss, Meanders and their application to lower bound arguments, J. Comp. Sys. Sci., 37 (1988), pp. 118-129. 
[5] B. Alpern, L. Carter, And E. Feig, Uniform memory hierarchies, in Proc. 31st Annual IEEE Symposium on Foundations of Computer Science, 1990, pp. 600-608.

[6] J. Balcázar, J. Díaz, And J. Gabarró, Structural Complexity I, Springer Verlag, 1988.

[7] D. M. Barrington, N. Immerman, and H. Straubing, On uniformity within $N C^{1}$, in Proc. 3rd Annual IEEE Conference on Structure in Complexity Theory, 1988, pp. 47-59.

[8] — On uniformity within $N C^{1}$, J. Comp. Sys. Sci., 41 (1990), pp. 274-306.

[9] A. Ben-Amram and Z. Galil, Lower bounds for data structure problems on RAMs, in Proc. 32nd Annual IEEE Symposium on Foundations of Computer Science, 1991, pp. 622-631.

[10] — On pointers versus addresses, J. Assn. Comp. Mach., 39 (1992), pp. 617-648.

[11] G. Blelloch, Vector Models for Data-Parallel Computing, MIT Press, 1990.

[12] M. Blum, A machine-independent theory of the complexity of recursive functions, J. Assn. Comp. Mach., 14 (1967), pp. 322-336.

[13] R. Book and S. Greibach, Quasi-realtime languages, Math. Sys. Thy., 4 (1970), pp. 97-111.

[14] A. Chandra, D. Kozen, and L. Stockmeyer, Alternation, J. Assn. Comp. Mach., 28 (1981), pp. 114-133.

[15] J. Chang, O. Ibarra, And A. Vergis, On the power of one-way communication, J. Assn. Comp. Mach., 35 (1988), pp. 697-726.

[16] J. Chen And C. YAP, Reversal complexity, SIAM J. Comput., 20 (1991), pp. 622-638.

[17] M. Conner, Sequential machines realized by group representations, Inform. and Comp., 85 (1990), pp. 183-201.

[18] S. Cook And R. Reckhow, Time bounded random access machines, J. Comp. Sys. Sci., 7 (1973), pp. 354-375.

[19] D. Coppersmith And S. Winograd, Matrix multiplication via arithmetical progressions, J. Symbolic Computation, 9 (1990), pp. 251-280.

[20] M. Dietzfelbinger, W. MaAss, and G. Schnitger, The complexity of matrix transposition on one-tape off-line Turing machines, Theor. Comp. Sci., 82 (1991), pp. 113-129.

[21] C. Elgot and A. Robinson, Random-access stored-program machines, J. Assn. Comp. Mach., 11 (1964), pp. 365-399.

[22] Y. Feldman And E. Shapiro, Spatial machines: A more-realistic approach to parallel computation, Comm. Assn. Comp. Mach., 35 (1992), pp. 60-73.

[23] P. Fischer, A. Meyer, And A. Rosenberg, Real-time simulations of multihead tape units, J. Assn. Comp. Mach., 19 (1972), pp. 590-607.

[24] M. Fürer, Data structures for distributed counting, J. Comp. Sys. Sci., 29 (1984), pp. 231-243.

[25] Z. Galil And J. Seiferas, Time-space optimal string matching, J. Comp. Sys. Sci., 26 (1983), pp. 280-294.

[26] E. Graedel, On the notion of linear-time computability, Intern. J. Found. Comp. Sci., 1 (1990), pp. 295-307.

[27] E. Grandjean, A natural NP-complete problem with a nontrivial lower bound, SIAM J. Comput., 17 (1988), pp. 786-809.

[28] - A nontrivial lower bound for an NP problem on automata, SIAM J. Comput., 19 (1990), pp. 438-451.

[29] E. GRANDJEAn AND J. Robson, RAM with compact memory: a robust and realistic model of computation, in CSL '90: Proceedings of the 4th Annual Workshop in Computer Science Logic, vol. 533 of Lect. Notes in Comp. Sci., Springer Verlag, 1991, pp. 195-233.

[30] Y. Gurevich and S. Shelah, Nearly-linear time, in Proceedings, Logic at Botik '89, vol. 363 of Lect. Notes in Comp. Sci., Springer Verlag, 1989, pp. 108-118.

[31] J. Hartmanis and R. Stearns, On the computational complexity of algorithms, Transactions of the AMS, 117 (1965), pp. 285-306.

[32] — Algebraic Structure Theory of Sequential Machines, Prentice-Hall, Englewood Cliffs, NJ, 1966.

[33] F. Hennie and R. Stearns, Two-way simulation of multitape Turing machines, J. Assn. Comp. Mach., 13 (1966), pp. 533-546.

[34] T. Heywood And S. Ranka, A practical hierarchical model of parallel computation I: The model, J. Par. Dist. Comp., 16 (1992), pp. 212-232.

[35] J.-W. Hong, On similarity and duality of computation I, Inform. and Comp., 62 (1985), pp. 109-128.

[36] J. Hopcroft and J. Ullman, Introduction to Automata Theory, Languages, and Computation, Addison-Wesley, Reading, MA, 1979.

[37] O. IbarRa, Systolic arrays: characterizations and complexity, in The Proceedings of the 1986 Conference on Mathematical Foundations of Computer Science, Lecture Notes in Computer Science No. 233, Springer Verlag, 1986, pp. 140-153.

[38] O. IbarRa And T. JiAnG, On one-way cellular arrays, SIAM J. Comput., 16 (1987), pp. 1135- 
1153.

[39] O. IBARRA AND S. KIM, Characterizations and computational complexity of systolic trellis automata, Theor. Comp. Sci., 29 (1984), pp. 123-153.

[40] O. Ibarra, S. KIM, ANd M. PALIS, Designing systolic algorithms using sequential machines, IEEE Transactions on Computing, 35 (1986), pp. 531-542.

[41] O. Ibarra, M. Palis, and S. Kim, Some results concerning linear iterative (systolic) arrays, J. Par. Dist. Comp., 2 (1985), pp. 182-218.

[42] T. Jiang And M. LI, $K$ one-way heads cannot do string-matching, in Proc. 25th Annual ACM Symposium on the Theory of Computing, 1993, pp. 62-70.

[43] T. Kameda And R. Vollmar, Note on tape reversal complexity of languages, Inform. and Control, 17 (1970), pp. 203-215.

[44] J. Katajainen, J. van Leeuwen, and M. Penttonen, Fast simulation of Turing machines by random access machines, SIAM J. Comput., 17 (1988), pp. 77-88.

[45] A. Kolmogorov and V. Uspenskit, On the definition of an algorithm, Uspekhi Mat. Nauk, 13 (1958), pp. 3-28. English transl. in Russian Math Surveys 30 (1963) 217-245.

[46] R. Kosaraju, Real-time simulation of concatenable double-ended queues by double-ended queues, in Proc. 11th Annual ACM Symposium on the Theory of Computing, 1979, pp. 346351.

[47] K. Krohn and J. Rhodes, Algebraic theory of machines. I. prime decomposition theorem for finite semigroups and machines, Trans. Amer. Math. Soc., 116 (1965), pp. 450-464.

[48] K. Krohn, J. Rhodes, And B. Tilson, The prime decomposition theorem of the algebraic theory of machines, in Algebraic Theory of Machines, Languages, and Semigroups, M. Arbib, ed., Academic Press, 1968. Ch. 5; see also chs. 4 and 6-9.

[49] D. Leivant, Descriptive characterizations of computational complexity, J. Comp. Sys. Sci., 39 (1989), pp. 51-83.

[50] M. Loui, Simulations among multidimensional Turing machines, Theor. Comp. Sci., 21 (1981), pp. $145-161$.

[51] - Optimal dynamic embedding of trees into arrays, SIAM J. Comput., 12 (1983), pp. 463472 .

[52] — Minimizing access pointers into trees and arrays, J. Comp. Sys. Sci., 28 (1984), pp. 359378.

[53] G. Manacher, Steady-paced-output and fractional-on-line algorithms on a RAM, Inf. Proc. Lett., 15 (1982), pp. 47-52.

[54] Y. Mansour, N. Nisan, AND P. TiWari, The computational complexity of universal hashing, Theor. Comp. Sci., 107 (1993), pp. 121-133.

[55] D. Muller and F. Preparata, Bounds to complexities of networks for sorting and switching, J. Assn. Comp. Mach., 22 (1975), pp. 195-201.

[56] W. Paul, N. Pippenger, E. Szemerédi, and W. Trotter, On determinism versus nondeterminism and related problems, in Proc. 24th Annual IEEE Symposium on Foundations of Computer Science, 1983, pp. 429-438.

[57] W. Paul and R. Reischuk, On time versus space II, J. Comp. Sys. Sci., 22 (1981), pp. 312-327.

[58] W. Paul, J. Seiferas, and J. Simon, An information-theoretic approach to time bounds for on-line computation, J. Comp. Sys. Sci., 23 (1981), pp. 108-126.

[59] N. Pippenger, On simultaneous resource bounds, in Proc. 20th Annual IEEE Symposium on Foundations of Computer Science, 1979, pp. 307-311.

[60] N. Pippenger and M. Fischer, Relations among complexity measures, J. Assn. Comp. Mach., 26 (1979), pp. 361-381.

[61] V. Pratt and L. Stockmeyer, A characterization of the power of vector machines, J. Comp. Sys. Sci., 12 (1976), pp. 198-221.

[62] K. REgAn, A new parallel vector model, with exact characterizations of $N C^{k}$, in Proc. 11th Annual Symposium on Theoretical Aspects of Computer Science, vol. 778 of Lect. Notes in Comp. Sci., Springer Verlag, 1994, pp. 289-300.

[63] R. Reischuk, A fast implementation of multidimensional storage into a tree storage, Theor. Comp. Sci., 19 (1982), pp. 253-266.

[64] W. Ruzzo, On uniform circuit complexity, J. Comp. Sys. Sci., 22 (1981), pp. 365-383.

[65] C. Schnorr, Satisfiability is quasilinear complete in NQL, J. Assn. Comp. Mach., 25 (1978), pp. 136-145.

[66] A. Schönhage, Storage modification machines, SIAM J. Comput., 9 (1980), pp. 490-508.

[67] — A nonlinear lower bound for random-access machines under logarithmic cost, J. Assn. Comp. Mach., 35 (1988), pp. 748-754.

[68] P. van Emde BoAs, Machine models and simulations, in Handbook of Theoretical Computer Science, J. V. Leeuwen, ed., vol. A, Elsevier and MIT Press, 1990, pp. 1-66. 
[69] D. Willard, A density control algorithm for doing insertions and deletions in a sequentially ordered file in a good worst-case time, Inform. and Comp., 97 (1992), pp. 150-204.

\section{Appendix: Proof of Theorem 2.1.}

For every move state $q$ in $M$ we add a new GST $S_{q}$ that performs a 1-bit empty pull just to read the currently-scanned character $d$, and then sends control to $\delta(q, d)$. This modification no more than doubles the $\mu$-access charges, and gives $M$ the following property: for any pass by a GST $S_{i}$, the next GST $S_{k}$ to be invoked (or HALT) is a function only of $i$ and the character $c$ that caused $S_{i}$ to exit, and there is at most one intervening move. Henceforth we assume that $M$ has this form, and number its GST chips by $S_{0}, \ldots, S_{r}$, with $S_{0}$ as start chip.

$M^{\prime}$ uses an alphabet $\Gamma^{\prime}$ which includes the alphabet $\Gamma$ of $M$, a surrogate blank @, tokens $\left\{s_{0}, \ldots, s_{r}\right\}$ for the chips of $M$, markers $\left\{m_{U}, m_{L}, m_{R}, m_{n o}, m_{H}\right\}$ for the three kinds of move, "no move," and HALT, special instruction markers $\left\{I_{0}, \ldots, I_{12}\right\}$, plus certain tuples of length up to 7 of the foregoing characters. We also use @ to indicate that the symbol written to cell 0 is immaterial.

During the simulation, the first component of every tuple in a cell $i$ is the character $c_{i} \in \Gamma$ in that cell of the tape of $M$. Except initially, cell 1 holds both $c_{0}$ and $c_{1}$, so that cell 0 can be overwritten by other characters. This also allows $M^{\prime}$ to simulate all moves by $M$ without ever moving its own cell- $a$ head back to cell 0 . The markers $I_{0}$ and $I_{1}$ tell $M^{\prime}$ when the cell- $a$ head of $M$ is in cell 0 or 1 . For $a \geq 2$, the heads of $M$ and $M^{\prime}$ coincide. The other main invariant of the simulation is that the only cell besides cells 0 and 1 to contain multiple symbols is cell $a$. The two initial moves of $M^{\prime}$ set up these invariants.

$$
\begin{aligned}
& \text { Character(s) read Action (Initial mode is } R a, a=0 \text {.) } \\
& \overline{c_{0}, c_{1}} \quad \overline{\text { Pull }\left[c_{0}, c_{1}\right] \text { to cell } 0, a:=a, \text { mode }}:=0 R \text {. } \\
& {\left[c_{0}, c_{1}\right], c_{1} \quad \text { Put @ into cell } 0 \text { and }\left[c_{1}, c_{0}, s_{0}, I_{0}\right] \text { into cell } 1, a:=2 a+1 \text {, }} \\
& \text { mode }:=\text { Ra. }
\end{aligned}
$$

The first move must automatically be executed every time $M^{\prime}$ moves its tape head to a new cell $a, a \geq 2$, since this cell and cell $a+1$ will always contain single characters over $\Gamma$. However, the second move is unique to the initialization because cell 1 will never again hold a single character. The cell- $a$ head of $M^{\prime}$ is now on cell 1 , but the $I_{0}$ enables $M$ to record that the cell- $a$ head of $M$ is still on cell 0 .

The lone GST $S$ of $M^{\prime}$ includes two copies of each GST $S_{i}$ of $M$. The first is a "dummy copy" which simulates $S_{i}$ but suppresses output until it picks up the character $c$ that causes $S_{i}$ to exit. On this exiting transition, the dummy outputs a token $s_{k}$ for the next GST $S_{k}$ and a token $m$ for the intervening move, or $m_{n o}$ for none, or $m_{H}$ for HALT. The other copy simulates the actual pass by $S_{i}$. It has special states that distinguish whether $S_{i}$ has written zero, one, or at least two output symbols in the pass, since the first one or two symbols of the output $y$ are altered. If $S_{i}$ performs a pull and $|y| \geq 2$, we define $c_{0}^{\prime}:=y_{0}$ if $y_{0} \neq B$, but $c_{0}^{\prime}:=c_{0}$ if $y_{0}=B$. Similarly $c_{1}^{\prime}:=y_{1}$ if $y_{1} \neq B$, but $c_{1}^{\prime}:=c_{1}$ if $y_{1}=B$. On the tape of $M^{\prime}$, the output $y$ looks like $\left[c_{0}^{\prime}, c_{1}^{\prime}, \ldots\right]\left[c_{1}^{\prime}, c_{0}^{\prime}, \ldots\right] y_{2} \cdots y_{l}$, where $l=|y|$. For $|y| \leq 1$, treat the missing $y_{1}$ and/or $y_{0}$ as $B$. Besides these functional conventions on $s_{k}, m, c_{0}^{\prime}$, and $c_{1}^{\prime}$, we omit reference to the address $a$ if it is not changed, and omit the second character read by $S$ when it does not affect control at the large initial branch. Let $S_{i}$ be the current GST of $M$. 


\begin{tabular}{|c|c|}
\hline Character(s) read & Action (Current mode is $R a, a=1$.) \\
\hline$\overline{\left[c_{1}, c_{0}, s_{i}, I_{0}\right]}$ & $\begin{array}{l}\text { By the validity conditions (Definition } 2.3 \text { ), the output } y \text { by } S_{i} \\
\text { has length at most } 2 \text {. Hence the next-move token } m \text { and next- } \\
\text { GST token } s_{k} \text { can be picked up and the output } y \text { written in } \\
\text { one pass, without needing the dummy copy of } S_{i} \text {. If } m=m_{H} \text {, } \\
S \text { pulls @ to cell } 0 \text { and }\left[c_{1}^{\prime}, c_{0}^{\prime}, I_{12}\right] \text { to cell } 1 \text {. If } m=m_{R} \text {, pulls } \\
@\left[c_{1}^{\prime}, c_{0}^{\prime}, s_{k}, I_{1}\right] \text { to signify that the cell- } a \text { head of } M \text { is now on } \\
\text { cell } 1 \text {. Else } S \text { pulls @ }\left[c_{1}^{\prime}, c_{0}^{\prime}, s_{k}, I_{0}\right] \text {, and this step repeats. In } \\
\text { each case, mode }:=R a \text {. }\end{array}$ \\
\hline$\left[c_{1}, c_{0}, I_{12}\right]$ & Pull $c_{0}$ into cell $0, c_{1}$ into cell 1 , and HaLT. \\
\hline$\left[c_{1}, c_{0}, s_{i}, I_{1}\right]$ & $\begin{array}{l}\text { Simulate } S_{i} \text { as for }\left[c_{1}, c_{0}, s_{i}, I_{0}\right] \text { to get } m, s_{k} \text {, and } y \text {, but } \\
\text { treat } c_{1} \text { as the first input character to } S_{i} \text {. If } m=m_{H} \text { pull } \\
@\left[c_{1}^{\prime}, c_{0}^{\prime}, I_{12}\right], \text { if } m=m_{U} \text { pull } @\left[c_{1}^{\prime}, c_{0}^{\prime}, s_{k}, I_{0}\right] \text {, and if } m=m_{n o} \text {, }{ }^{\prime} \text {, In } \\
\text { pull } @\left[c_{1}^{\prime}, c_{0}^{\prime}, s_{k}, I_{1}\right] \text {. In these three cases, the address of } M^{\prime} \\
\text { stays at } 1 \text {. If } m=m_{L} \text {, then pull @ }\left[c_{1}^{\prime}, c_{0}^{\prime}, s_{k}\right] \text { and effect } a:=2 a \text {. } \\
\text { If } m=m_{R} \text {, pull @ }\left[c_{1}^{\prime}, c_{0}^{\prime}, s_{k}\right] \text { and effect } a:=2 a+1 \text {. In every } \\
\text { case, the mode stays } R a \text {. }\end{array}$ \\
\hline
\end{tabular}

The last two cases give $a \geq 2$. When $a \geq 2$, the next pass by $S$ encounters a single character $c_{a} \in \Gamma$ on its start transition (possibly $c_{a}=B$ ), and $S$ must perform the first operation above. This overwrites the @ in cell 0. However, the new character $\left[c_{1}^{\prime}, c_{0}^{\prime}, s_{k}\right]$ in cell 1 prevents the initial sequence from recurring, viz.:

Character(s) read

$c_{a}, c_{a+1}$

$B$

$\left[c_{a}, c_{a+1}\right],\left[c_{1}, c_{0}, s_{i}\right]$

$\left[c_{a}, c_{0}, c_{1}, s_{i}, I_{2}\right]$
Action (Current mode is $R a, a \geq 2$.)

$\overline{\text { Pull }\left[c_{a}, c_{a+1}\right] \text { to cell } 0, a:=a, \text { mode }}:=0 R$.

Pull $\left[@, @, I_{0}\right]$ to cell $0, a:=a, \operatorname{mode}:=0 R$.

Put $\left[c_{a}, c_{0}, c_{1}, s_{i}, I_{2}\right]$ into cell $a$. If the label of $S_{i}$ is $L a$ then mode $:=$ La, else mode $:=R a$.

If $S_{i}$ is labeled $0 L$ or $0 R$, then pull $\left[c_{0}, c_{1}, c_{a}, s_{i}, I_{6}\right]$ into cell 0 , and mode $:=$ the mode of $S_{i}$. Else $S$ simulates the dummy copy of $S_{i}$ to find $m$ and $s_{k}$, treating $c_{a}$ as the first input character to $S_{i}$, and pulls $\left[c_{0}, c_{1}, c_{a}, m, s_{k}, s_{i}, I_{3}\right]$ to cell 0 with mode $:=0 R$.

$\left[c_{0}, c_{1}, c_{a}, m, s_{k}, s_{i}, I_{3}\right]$ Put $\left[c_{a}, c_{0}, c_{1}, m, s_{k}, s_{i}, I_{4}\right]$ into cell $a$, mode $:=$ the mode of $S_{i}$.

$\left[c_{a}, c_{0}, c_{1}, m, s_{k}, s_{i}, I_{4}\right]$ Simulate the pull move by $S_{i}$, translating its output $y$ to $\left[c_{0}^{\prime}, c_{1}^{\prime}, c_{a}, m, I_{5}\right]\left[c_{1}^{\prime}, c_{0}^{\prime}, s_{k}\right] y_{2} \cdots y_{l}$, and change mode to $0 R$. Remark: For $c_{a}$ to be correct, it is vital that cell $a$ not be overwritten in this pull.

$\left[c_{0}, c_{1}, c_{a}, m, I_{5}\right] \quad$ Put $c_{a}$ into cell $a$. On exit, if $m=m_{n o}$ then leave $a$ unchanged, if $m=m_{U}$ effect $a:=\lfloor a / 2\rfloor$, if $m=m_{L}$ effect $a:=2 a$, and if $m=m_{R}$ effect $a:=2 a+1$. In each of these four cases, mode $:=R a$. For $m=m_{H}$, see below.

If the last move was $u p$, i.e. $a$ to $\lfloor a / 2\rfloor$, we may now have $a=1$ again. Since the "sentinel" in cell 1 is always correctly updated to the next GST $S_{i}$, this is handled by:

$\left[c_{1}, c_{0}, s_{i}\right] \quad$ Same as for $\left[c_{1}, c_{0}, s_{i}, I_{1}\right]$.

If still $a \geq 2$, then $S$ once again senses single characters in cells $a$ and $a+1$, and the cycle repeats. The other branch with instruction 6 goes: 
$\left[c_{0}, c_{1}, c_{a}, s_{i}, I_{6}\right] \quad$ Here $S_{i}$ is labeled $0 L$ or $0 R$, and this is the current mode. $S$ treats $c_{0}, c_{1}$ as the first two input characters in simulating the dummy copy of $S_{i}$, and puts $\left[c_{a}, c_{0}, c_{1}, m, s_{k}, s_{i}, I_{7}\right]$ into cell $a$ with mode $:=R a$.

$\left[c_{a}, c_{0}, c_{1}, m, s_{k}, s_{i}, I_{7}\right]$ Pull $\left[c_{0}, c_{1}, c_{a}, m, s_{k}, s_{i}, I_{8}\right]$ into cell 0, mode $:=$ the mode of $S_{i}$.

$\left[c_{0}, c_{1}, c_{a}, m, s_{k}, s_{i}, I_{8}\right]$ Simulate the put by $S_{i}$. If the output $y$ is empty or begins with $B$, let $c_{a}^{\prime}:=c_{a}$. Else let $c_{a}^{\prime}:=y_{0}$. Copy $y$ as $\left[c_{a}^{\prime}, c_{0}, c_{1}, m, s_{k}, I_{9}\right]$, and set mode $:=0 R$.

$\left[c_{a}, c_{0}, c_{1}, m, s_{k}, I_{9}\right] \quad$ Pull $\left[c_{0}, c_{1}, c_{a}, m, I_{5}\right]$ to cell 0 and $\left[c_{1}, c_{0}, s_{k}\right]$ to cell 1 , mode $:=$ $R a$.

The validity conditions prevent cell $a$ from being overwritten in a pull. It is possible for cell 1 to be overwritten by a leftward put that exits after just one input bit, but this can only happen if $a \leq C$, where $C$ is the maximum number of bits a leftward pull chip of $M$ can write in its first transition. The problem can be solved either by exploiting the ability of $M$ itself to remember $C$-many characters in its finite control, or by reprogramming $M$ so that no leftward pull chip outputs more than one symbol on its first step. Details are left to the reader.

The final halting routine involves a "staircase" to leave the tape exactly the same as that of $M$ at the end of the computation. It picks up in the case $\left[c_{0}, c_{1}, c_{a}, m, I_{5}\right]$ with $m=m_{H}$.

$\left[c_{0}, c_{1}, c_{a}, m_{H}, I_{5}\right] \quad$ Put $\left[c_{a}, c_{0}, c_{1}, I_{10}\right]$ into cell $a$, mode $:=R a$.

$\left[c_{a}, c_{0}, c_{1}, I_{10}\right] \quad$ Pull $\left[c_{0}, c_{1}, c_{a}, I_{11}\right]$ to cell 0 and $\left[c_{1}, c_{0}, I_{12}\right]$ to cell 1 , with mode $:=0 R$.

$\left[c_{0}, c_{1}, c_{a}, I_{11}\right] \quad$ Put $c_{a}$ into cell $a$, effect $a:=\lfloor a / 2\rfloor$, mode $:=R a$.

$c_{a}, c_{a+1} \quad$ Pull $\left[c_{a}, c_{a+1}\right]$ to cell 0, mode $:=0 R$.

$\left[c_{a}, c_{a+1}\right],\left[c_{1}, c_{0}, I_{12}\right] \quad$ Put $c_{a}$ into cell $a$, effect $a:=\lfloor a / 2\rfloor$, mode $:=R a$.

$\left[c_{1}, c_{0}, I_{12}\right] \quad$ As above, pull $c_{0}$ into cell $0, c_{1}$ into cell 1 , and HALt.

$M^{\prime}$ uses exactly the same tape cells as $M$, making at most eight passes of equal or less cost for each pass by $M$. The final "staircase" down from cell $a$ is accounted against the $\mu$-charges for $M$ to have moved out to cell $a$. Hence both the number of bits processed by $M^{\prime}$ and the $\mu$-acc charges to $M^{\prime}$ are within a constant factor of their counterparts in $M$.

For the converse simulation of the reduced form $S$ by a BM $M$, the only technical difficulty is that $S$ may have different exiting transitions on the same character $c$. The solution is to run a dummy copy of $S$ that outputs a token $t$ for the state in which $S$ exits. Then $t$ is used to send control to the move state of $M^{\prime}$ that corresponds to the label $l_{1}(t)$, and thence to a copy of $S$ with the pass-type label $l_{2}(t)$. The details of running the dummy copy are the same as above. $\square$

By using more "instruction markers" one can make the mode of $M^{\prime}$ always follow the cycle $R a, 0 R, L a, 0 L$. Hence the only decision that need depend on the terminal state of the lone GST $S$ is the next origin cell $a$. 